\title{
Interior Schauder Estimates for Elliptic Equations Associated with Lévy Operators
}

\section{Franziska Kühn ${ }^{1}$}

Received: 24 April 2020 / Accepted: 19 November 2020 / Published online: 23 January 2021

(C) The Author(s) 2021

\section{Abstract}

We study the local regularity of solutions $f$ to the integro-differential equation

$$
A f=g \text { in } U
$$

for open sets $U \subseteq \mathbb{R}^{d}$, where $A$ is the infinitesimal generator of a Lévy process $\left(X_{t}\right)_{t \geq 0}$. Under the assumption that the transition density of $\left(X_{t}\right)_{t \geq 0}$ satisfies a certain gradient estimate, we establish interior Schauder estimates for both pointwise and weak solutions $f$. Our results apply for a wide class of Lévy generators, including generators of stable Lévy processes and subordinated Brownian motions.

Keywords Lévy process · Integro-differential equation · Schauder estimate · Hölder space · Gradient estimate

Mathematics Subject Classification (2010) Primary 60G51 · Secondary 45K05, 60J35

\section{Introduction}

Let $\left(X_{t}\right)_{t \geq 0}$ be a $d$-dimensional Lévy process. By the Lévy-Khintchine formula, $\left(X_{t}\right)_{t \geq 0}$ is uniquely characterized (in distribution) by its infinitesimal generator $A$, which is an integrodifferential operator with representation

$$
\begin{aligned}
A f(x)= & b \cdot \nabla f(x)+\frac{1}{2} \nabla \cdot Q \nabla f(x) \\
& +\int_{\mathbb{R}^{d} \backslash\{0\}}\left(f(x+y)-f(x)-\nabla f(x) \cdot y \mathbb{1}_{(0,1)}(|y|)\right) v(d y)
\end{aligned}
$$

for $f \in C_{c}^{\infty}\left(\mathbb{R}^{d}\right)$, here $(b, Q, v)$ denotes the Lévy triplet of $\left(X_{t}\right)_{t \geq 0}$, cf. Section 2 . In this paper, we study the local Hölder regularity of weak and pointwise solutions $f$ to the integro-differential equation

$$
A f=g \quad \text { in } U
$$

Franziska Kühn

franziska.kuehn1@tu-dresden.de

1 Fakultät Mathematik, Institut für Mathematische Stochastik, TU Dresden,

Dresden, 01062, Germany 
for open sets $U \subseteq \mathbb{R}^{d}$. We are interested in interior Schauder estimates, i.e. our aim is to describe the regularity of $f$ on the set $\left\{x \in U ; d\left(x, U^{c}\right)>\delta\right\}$ for $\delta>0$ and to establish estimates for its Hölder norm. We will see that there is a close connection between the regularity of $f$ and "volatility" of the Lévy process: the higher the "volatility" (caused by a non-vanishing diffusion component or a high small-jump activity), the higher the regularity of $f$.

In the absence of a jump part, i.e. if $v=0$, the generator $A$ is a second-order differential operator and interior Schauder estimates for solutions to $A f=g$ are well studied, see e.g. Gilbarg \& Trudinger [8]. One of the most prominent non-local Lévy operators is the fractional Laplacian $-(-\Delta)^{\alpha / 2}, \alpha \in(0,2)$, defined by

$$
-(-\Delta)^{\alpha / 2} f(x)=c_{d, \alpha} \int_{y \neq 0}\left(f(x+y)-f(x)-\nabla f(x) \cdot y \mathbb{1}_{(0,1)}(|y|)\right) \frac{1}{|y|^{d+\alpha}} d y
$$

for some normalizing constant $c_{d, \alpha}>0$. The fractional Laplacian is the infinitesimal generator of the isotropic $\alpha$-stable Lévy process and plays an important role in analysis and probability theory, see e.g. the survey paper [23] for a detailed discussion. Global regularity estimates for solutions to $-(-\Delta)^{\alpha / 2} f=g$ go back to Stein [36], see also Bass [1]. Since then, several extensions and refinements of these estimates have been obtained. Ros-Oton $\&$ Serra [29] studied the interior Hölder regularity of solutions to equations $A f=g$ for symmetric $\alpha$-stable operators $A$ under a mild degeneracy condition on the spectral measure. In the recent paper [18], global Schauder estimates

$$
\|f\|_{\mathcal{C}_{b}^{\alpha+\kappa}\left(\mathbb{R}^{d}\right)} \leq c\left(\|f\|_{\infty}+\|g\|_{\mathcal{C}_{b}^{\kappa}\left(\mathbb{R}^{d}\right)}\right), \quad \kappa \geq 0,
$$

were obtained for a wide class of Lévy processes satisfying a certain gradient estimate, see (C2) below; here $\mathcal{C}_{b}^{\beta}\left(\mathbb{R}^{d}\right)$ denotes the Hölder-Zygmund space of order $\beta$, cf. Section 2 for the definition. Moreover, there are numerous results on the regularity of functions which are harmonic with respect to a Lévy generator, see e.g. [9, 10, 21, 22, 24, 37]. Let us mention that the regularity of solutions to integro-differential equations $A f=g$ has been studied, in greater generality, for classes of Lévy-type operators, see e.g. [1, 5, 12, 14, 20, 25, 27]. Of course, Schauder estimates are also of interest for parabolic equations, we point the interested reader to the recent works $[4,11,26,39]$ and the references therein.

In this paper, we combine the global Schauder estimates from [18], cf. Eq. 1, with a truncation technique to derive local Hölder estimates for solutions to $A f=g$. We will assume that the Lévy process $\left(X_{t}\right)_{t \geq 0}$ with Lévy triplet $(b, Q, v)$ satisfies the following conditions.

(C1) The characteristic exponent $\psi$ satisfies the Hartman-Wintner growth condition

$$
\lim _{|\xi| \rightarrow \infty} \frac{|\operatorname{Re} \psi(\xi)|}{\log (1+|\xi|)}=\infty .
$$

(C2) There exist constants $M>0$ and $\alpha \in(0,2]$ such that the transition density $p_{t}, t>0$, satisfies the gradient estimate

$$
\int_{\mathbb{R}^{d}}\left|\nabla p_{t}(x)\right| d x \leq M t^{-1 / \alpha}, \quad t \in(0,1) .
$$

(C3) Either $\alpha>1$ or $Q=0$. Moreover, $\alpha+1>\gamma$ for a constant $\gamma \in(0,2]$ with $\int_{|y| \leq 1}|y|^{\gamma} v(d y)<\infty$.

The Hartman-Wintner condition (C1) implies that the law of $X_{t}, t>0$, has a density $p_{t} \in$ $C_{b}^{\infty}\left(\mathbb{R}^{d}\right)$ with respect to Lebesgue measure, see [17] for a detailed discussion. Condition 
(C3) is essentially a balance condition on the growth of $\operatorname{Re} \psi$ at infinity, see the final part of Section 4 for details. If $\alpha>1$, we may choose $\gamma=2$ in (C3) since $\int_{|y| \leq 1}|y|^{2} v(d y)<\infty$ holds for any Lévy measure. The gradient estimate $(\mathrm{C} 2)$ is equivalent to

$$
\left\|\nabla P_{t} f\right\|_{\infty} \leq C t^{-1 / \alpha}\|f\|_{\infty}, \quad t \in(0,1), f \in \mathcal{B}_{b}\left(\mathbb{R}^{d}\right),
$$

where $P_{t} f(x)=\mathbb{E} f\left(x+X_{t}\right)$ is the semigroup of $\left(X_{t}\right)_{t \geq 0}$. The constant $\alpha$ is always less or equal than 2; this follows from the fact that the growth condition $|\psi(\xi)| \leq c\left(1+|\xi|^{\varrho}\right)$, $\xi \in \mathbb{R}^{d}$, implies $\alpha \leq \varrho$, cf. [18, Remark 3.2(ii)].

Next we state our main results; see Section 2 for the definition of the notation used in the statements.

Theorem 1 Let $\left(X_{t}\right)_{t \geq 0}$ be a Lévy process with weak infinitesimal generator $(A, \mathcal{D}(A))$ satisfying $(\mathrm{C} 1)-(\mathrm{C} 3)$, and denote by $\alpha \in(0,2]$ the constant from $(\mathrm{C} 2)$. Let $f$ be a weak solution to the equation

$$
\text { Af }=g \text { in } U
$$

for an open set $U \subseteq \mathbb{R}^{d}$. Set $U_{\delta}:=\left\{x \in U ; d\left(x, U^{c}\right)>\delta\right\}$ for $\delta>0$.

i). If $f \in L^{\infty}\left(\mathbb{R}^{d}\right)$ and $g \in L^{\infty}(U)$, then $f$ has a modification $\tilde{f}$ which is continuous on $U$ and satisfies the interior Schauder estimate

$$
\|\tilde{f}\|_{\mathcal{C}_{b}^{\alpha}\left(U_{\delta}\right)} \leq C_{\delta}\left(\|f\|_{L^{\infty}\left(\mathbb{R}^{d}\right)}+\|g\|_{L^{\infty}(U)}\right)
$$

for every $\delta>0$ with $U_{\delta} \neq \emptyset$. The constant $C_{\delta}$ does not depend on $f, g$.

ii). If $f \in \mathcal{C}_{b}^{\kappa}\left(\mathbb{R}^{d}\right)$ and $g \in \mathcal{C}_{b}^{\kappa}(U)$ for some $\kappa>0$, then there exists for every $\delta>0$ with $U_{\delta} \neq \emptyset$ a constant $C_{\delta}>0$ (independent of $f, g$ ) such that

$$
\|f\|_{\mathcal{C}_{b}^{\kappa+\alpha}\left(U_{\delta}\right)} \leq C_{\delta}\left(\|f\|_{\mathcal{C}_{b}^{\kappa}\left(\mathbb{R}^{d}\right)}+\|g\|_{\mathcal{C}_{b}^{\kappa}(U)}\right) .
$$

Theorem 1 applies for a wide class of Lévy processes. It generalizes the interior Schauder estimates for stable processes obtained in [29], and for the particular case that there is no jump part, i.e. $v=0$, we recover the classical regularity estimates for secondorder differential operators with constant coefficients, see Section 4 for details and further examples.

Remark 1 i). Any weak solution $f \in L^{\infty}\left(\mathbb{R}^{d}\right)$ to $A f=g$ is only determined up to a Lebesgue null set. The interior Schauder estimate (3) implies continuity of $\tilde{f}$ on $U$, and so Eq. 3 can hold only for a suitably chosen representative $\tilde{f}$.

ii). The Hölder-Zygmund space $\mathcal{C}_{b}^{\beta}(U)$ and the classical Hölder space $C_{b}^{\beta}(U)$ coincide if $\beta$ is not a natural number. In consequence, Theorem 1 remains valid for the classical Hölder spaces if $\alpha \notin \mathbb{N}, \kappa \notin \mathbb{N}$ and $\alpha+\kappa \notin \mathbb{N}$. If $\alpha, \kappa$ or $\alpha+\kappa$ is a natural number, then it is crucial that we use Hölder-Zygmund spaces rather than classical Hölder spaces to describe the regularity of $f$. Consider, for instance, $U=\mathbb{R}^{d}$ and a Cauchy process $\left(X_{t}\right)_{t \geq 0}$, then $(\mathrm{C} 1)-(\mathrm{C} 3)$ hold for $\alpha=1$. If $f$ is a function in the domain of the strong infinitesimal generator $A$ of the Cauchy process $\left(X_{t}\right)_{t \geq 0}$, then Eq. 3 holds with $\tilde{f}=f$ and $g=A f$, i.e.

$$
\|f\|_{\mathcal{C}_{b}^{1}\left(\mathbb{R}^{d}\right)} \leq C\left(\|f\|_{\infty}+\|A f\|_{\infty}\right) .
$$

On the other hand, it is known that a function $f$ in the domain of the strong generator need not be differentiable, cf. [18], and so

$$
\|f\|_{C_{b}^{1}\left(\mathbb{R}^{d}\right)} \leq C\left(\|f\|_{\infty}+\|A f\|_{\infty}\right)
$$


fails to hold. This means that measuring the smoothness of $f$ using classical Hölder spaces gives suboptimal results: the best one can hope for is $f \in C_{b}^{1-\varepsilon}\left(\mathbb{R}^{d}\right), \varepsilon>0$, which is weaker than our statement $f \in \mathcal{C}_{b}^{1}\left(\mathbb{R}^{d}\right)$. This phenomenon can be also observed in [29], which studies the interior regularity of solutions to $A f=g$ for stable operators $A$ using classical Hölder spaces. Theorem 1 improves the results from [29] for stable operators with index of stability $\alpha=1$.

iii). In the proof of Theorem 1 we need (C1), (C2) only to be able to apply [18, Theorem 1.1], which gives the global Schauder estimate

$$
\|f\|_{\mathcal{C}_{b}^{\alpha}\left(\mathbb{R}^{d}\right)} \leq c\left(\|f\|_{\infty}+\|A f\|_{\infty}\right), \quad f \in C_{b}^{2}\left(\mathbb{R}^{d}\right) .
$$

Consequently, the interior Schauder estimates in Theorem 1 hold for any Lévy process $\left(X_{t}\right)_{t \geq 0}$ with generator $(A, \mathcal{D}(A))$ satisfying (C3) and the global Schauder estimate (5). This means that (the proof of) Theorem 1 actually gives a general procedure to localize Schauder estimates.

iv). Roughly speaking, the constant $\alpha$ from (C2) is a measure for the "volatility" of the Lévy process. Intuitively, if $\alpha>0$ is small, then the process has a vanishing diffusion part and few small jumps, i.e. the sample paths have a relatively high regularity (in the sense of Besov regularity, see [32] for some results on this topic); conversely, if $\alpha$ is close to 2 , then $\left(X_{t}\right)_{t \geq 0}$ has many small jumps or a non-vanishing diffusion part, and the sample paths are more irregular.

Theorem 1 shows that a high volatility of the Lévy process $\left(X_{t}\right)_{t \geq 0}$ results in a high regularity of $\left.f\right|_{U}$. This is a natural result, and we believe the regularity estimates to be optimal for many Lévy processes. In some cases, certain properties of the Lévy process or the Lévy triplet may lead to an additional smoothing effect; for instance, Grzywny \& Kwaśnicki [9, Theorem 1.7] studied the regularity of harmonic functions $f$ (i.e. $A f=0$ ) associated with unimodal Lévy processes and showed that the regularity of the density of the Lévy measure $v$ carries over to $f$; this regularity of $f$ is not related to the volatility of the process.

v). The assumption $f \in \mathcal{C}_{b}^{\kappa}\left(\mathbb{R}^{d}\right)$ in Theorem 1.ii) cannot be relaxed to $f \in \mathcal{C}_{b}^{\kappa}(U)$; for stable processes a counterexample can be found in [29, Proposition 6.1].

vi). If $f$ is in the domain of the strong infinitesimal generator of $\left(X_{t}\right)_{t \geq 0}$, then [18, Theorem 1.1] gives $f \in \mathcal{C}_{b}^{\alpha}\left(\mathbb{R}^{d}\right)$, and so the assumption $f \in \mathcal{C}_{b}^{\kappa}\left(\mathbb{R}^{d}\right)$ in Theorem 1.ii) is automatically satisfied for $\kappa \leq \alpha$, see also Corollary 2 below.

Our second main result gives interior Schauder estimates for pointwise solutions to the equation $A f=g$.

Corollary 1 Let $\left(X_{t}\right)_{t \geq 0}$ be a Lévy process satisfying $(\mathrm{C} 1)-(\mathrm{C} 3)$, and let $U \subseteq \mathbb{R}^{d}$ be an open set. Let $f \in \mathcal{B}_{b}\left(\mathbb{R}^{d}\right)$ be a function such that

$$
g(x):=\lim _{t \rightarrow 0} \frac{\mathbb{E} f\left(x+X_{t}\right)-f(x)}{t}
$$

exists for all $x \in U$ and assume that

$$
\sup _{x \in K} \sup _{t \in(0,1)}\left|\frac{\mathbb{E} f\left(x+X_{t}\right)-f(x)}{t}\right|<\infty
$$

for every compact set $K \subseteq U$. Denote by $\alpha \in(0,2]$ the constant from (C2), and define $U_{\delta}:=\left\{x \in U ; d\left(x, U^{c}\right)>\delta\right\}$ for $\delta>0$. 
i). If $g \in L^{\infty}(U)$ then there exists for every $\delta>0$ with $U_{\delta} \neq \emptyset$ a constant $C_{\delta}>0$ (independent of $f, g$ ) such that

$$
\|f\|_{\mathcal{C}_{b}^{\alpha}\left(U_{\delta}\right)} \leq C_{\delta}\left(\|f\|_{L^{\infty}\left(\mathbb{R}^{d}\right)}+\|g\|_{L^{\infty}(U)}\right) .
$$

ii). If $f \in \mathcal{C}_{b}^{\kappa}\left(\mathbb{R}^{d}\right)$ and $g \in \mathcal{C}_{b}^{\kappa}(U)$ for some $\kappa>0$ then there exists for every $\delta>0$ with $U_{\delta} \neq \emptyset$ a constant $C_{\delta}>0$ (independent of $f, g$ ) such that

$$
\|f\|_{\mathcal{C}_{b}^{\kappa+\alpha}\left(U_{\delta}\right)} \leq C_{\delta}\left(\|f\|_{\mathcal{C}_{b}^{\kappa}\left(\mathbb{R}^{d}\right)}+\|g\|_{\mathcal{C}_{b}^{\kappa}(U)}\right) .
$$

If $\left(X_{t}\right)_{t \geq 0}$ is transient, then the pointwise solution to the equation $A f=g$ is given by $f(x)=\int_{\mathbb{R}^{d}} G(x, y) g(y) d y$, where $G$ is the Green function, and so it is possible to understand Corollary 1 as a regularity result for the integrated Green function.

As an immediate consequence of Corollary 1, we obtain local Schauder estimates for functions in the domain in the strong infinitesimal generator. They extend in a natural way the global Schauder estimates from [18].

Corollary 2 Let $\left(X_{t}\right)_{t \geq 0}$ be a Lévy process satisfying $(\mathrm{C} 1)-(\mathrm{C} 3)$, and denote by $\alpha \in(0,2]$ the constant from $(\mathrm{C} 2)$. Let $f$ be a function in the domain of the strong infinitesimal generator, i.e. $f$ is a continuous function vanishing at infinity and the limit

$$
A f(x):=\lim _{t \rightarrow 0} \frac{\mathbb{E} f\left(x+X_{t}\right)-f(x)}{t}
$$

exists uniformly in $x \in \mathbb{R}^{d}$.

i). For each $\delta>0$ there exists a finite constant $C_{\delta}$ (not depending on $f$ ) such that

$$
\|f\|_{\mathcal{C}_{b}^{\alpha}(B(x, \delta))} \leq C_{\delta}\left(\|f\|_{\infty}+\|A f\|_{\infty, B(x, 2 \delta)}\right)
$$

for all $x \in \mathbb{R}^{d}$.

ii). If Af $\in \mathrm{e}_{b}^{\kappa}\left(\mathbb{R}^{d}\right)$ for some $\kappa>0$, then $f \in \mathcal{C}_{b}^{\kappa+\alpha}\left(\mathbb{R}^{d}\right)$ and

$$
\|f\|_{\mathcal{C}_{b}^{\alpha+\kappa}(B(x, \delta))} \leq C_{\delta}\left(\|f\|_{\infty}+\|A f\|_{\infty}+\|A f\|_{\mathcal{C}_{b}^{\kappa}(B(x, 2 \delta))}\right), \quad x \in \mathbb{R}^{d}, \delta>0,
$$

for a finite constant $C_{\delta}$, which does not depend on $f, g$.

The proof of Corollary 2 shows that the interior Schauder estimates i),ii) actually hold for any Lévy process $\left(X_{t}\right)_{t \geq 0}$ with generator $(A, \mathcal{D}(A))$ satisfying $(\mathrm{C} 3)$ and the global Schauder estimate

$$
\|f\|_{\mathcal{C}_{b}^{\alpha}\left(\mathbb{R}^{d}\right)} \leq c\left(\|f\|_{\infty}+\|A f\|_{\infty}\right), \quad f \in C_{b}^{2}\left(\mathbb{R}^{d}\right)
$$

see Remark 1.iii).

This paper is organized as follows. In Section 2 we introduce the basic definitions and some notation. Our main results are proved in Section 3, and examples are presented in Section 4. 


\section{Definitions}

We consider the Euclidean space $\mathbb{R}^{d}$ with the canonical scalar product $x \cdot y:=\sum_{j=1}^{d} x_{j} y_{j}$ and the Borel $\sigma$-algebra $\mathcal{B}\left(\mathbb{R}^{d}\right)$ generated by the open balls $B(x, r)$. If $f$ is a real-valued function, then supp $f$ denotes its support and $\nabla f$ the gradient of $f$. For $\beta \geq 0$ we set

$$
\lfloor\beta\rfloor:=\max \left\{k \in \mathbb{N}_{0} ; k \leq \beta\right\} .
$$

Function Spaces $\mathcal{B}_{b}\left(\mathbb{R}^{d}\right)$ denotes the space of bounded Borel measurable functions $f: \mathbb{R}^{d} \rightarrow \mathbb{R}$. The smooth functions with compact support are denoted by $C_{c}^{\infty}\left(\mathbb{R}^{d}\right)$. Superscripts $k \in \mathbb{N}$ are used to denote the order of differentiability, e.g. $f \in C_{b}^{k}\left(\mathbb{R}^{d}\right)$ means that $f$ and its derivatives up to order $k$ are bounded continuous functions. For $U \subseteq \mathbb{R}^{d}$ we set

$$
\|f\|_{\infty, U}:=\sup _{x \in U}|f(x)| \text { and }\|f\|_{\infty}:=\|f\|_{\infty, \mathbb{R}^{d}} .
$$

For every $\beta \geq 0$ and every open set $U \subseteq \mathbb{R}^{d}$ we define the Hölder-Zygmund space $\mathcal{C}_{b}^{\beta}(U)$ by

$$
\mathcal{C}_{b}^{\beta}(U):=\left\{f \in C_{b}(U) ;\|f\|_{\mathcal{C}_{b}^{\beta}(U)}:=\sup _{x \in U}|f(x)|+\sup _{x \in U} \sup _{0<|h|<\min \left\{1, r_{x} / k\right\}} \frac{\left|\Delta_{h}^{k} f(x)\right|}{|h|^{\beta}}<\infty\right\}
$$

where $r_{x}:=d\left(x, U^{c}\right)$ is the distance of $x$ from the complement of $U, k=\lfloor\beta\rfloor+1$ is the smallest natural number strictly larger than $\beta$ and

$$
\Delta_{h} f(x):=f(x+h)-f(x) \quad \Delta_{h}^{\ell} f(x):=\Delta_{h}\left(\Delta_{h}^{\ell-1} f\right)(x), \quad \ell \geq 2
$$

are iterated difference operators. For $U=\mathbb{R}^{d}$ it is known that replacing $k$ by an arbitrary number $j$ strictly larger than $\beta$ gives an equivalent norm, i.e.

$$
\|f\|_{\mathcal{C}_{b}^{\beta}\left(\mathbb{R}^{d}\right)}=\|f\|_{\infty}+\sup _{x \in \mathbb{R}^{d}} \sup _{0<|h|<1} \frac{\left|\Delta_{h}^{k} f(x)\right|}{|h|^{\beta}} \asymp\|f\|_{\infty}+\sup _{x \in \mathbb{R}^{d}} \sup _{0<|h|<1} \frac{\left|\Delta_{h}^{j} f(x)\right|}{|h|^{\beta}},
$$

cf. [38, Theorem 2.7.2.2]. We need a localized version of this result.

Lemma 1 Let $\beta \in(0, \infty)$, and let $U \subseteq \mathbb{R}^{d}$ be open. The following statements hold for any $j \geq k:=\lfloor\beta\rfloor+1$ :

i). There exists a constant $c>0$ such that

$$
\sup _{0<|h| \leq r} \frac{\left|\Delta_{h}^{k} f(x)\right|}{|h|^{\beta}} \leq c r^{-\beta}\|f\|_{\infty, U}+c \sup _{0<|h| \leq r / j} \sup _{z \in B(x, r(k+1))} \frac{\left|\Delta_{h}^{j} f(z)\right|}{|h|^{\beta}}
$$

for all $f \in C_{b}(U), r>0$, and $x \in U$ with $B(x, r(k+2)) \subseteq U$.

ii). If $\beta>1$ then there exists a constant $c>0$ such that

$$
|\nabla f(x)| \leq c r^{-\beta}\|f\|_{\infty, U}+c \sup _{0<|h| \leq r / j} \sup _{z \in B(x, r(k+1))} \frac{\left|\Delta_{h}^{j} f(z)\right|}{|h|^{\beta}}
$$

for all $f \in C_{b}^{1}(U), r>0$ and $x \in U$ with $B(x, r(k+2)) \subseteq U$.

We defer the proof of Lemma 1 to the Appendix. For $\beta \in(0, \infty) \backslash \mathbb{N}$ the Hölder-Zygmund space $\mathcal{C}_{b}^{\beta}\left(\mathbb{R}^{d}\right)$ coincides with the "classical" Hölder space $C_{b}^{\beta}\left(\mathbb{R}^{d}\right)$, cf. [38, Theorem 2.7.2.1]. If $\beta=k \in \mathbb{N}$, then the inclusion $C_{b}^{k}\left(\mathbb{R}^{d}\right) \subseteq \mathrm{C}_{b}^{k}\left(\mathbb{R}^{d}\right)$ is strict. 
Lévy Processes Let $(\Omega, \mathcal{A}, \mathrm{P})$ be a probability space. A stochastic process $X_{t}: \Omega \rightarrow \mathbb{R}^{d}$, $t \geq 0$, is a ( $d$-dimensional) Lévy process if $X_{0}=0$ almost surely, $\left(X_{t}\right)_{t \geq 0}$ has independent and stationary increments and $t \mapsto X_{t}(\omega)$ is right-continuous with finite left-hand limits for almost all $\omega \in \Omega$. The Lévy-Khintchine formula shows that every Lévy process is uniquely determined in distribution by its characteristic exponent $\psi: \mathbb{R}^{d} \rightarrow \mathbb{C}$ satisfying

$$
\mathbb{E} \exp \left(i \xi \cdot X_{t}\right)=\exp (-t \psi(\xi)), \quad t \geq 0, \xi \in \mathbb{R}^{d} .
$$

The characteristic exponent $\psi$ has a Lévy-Khintchine representation

$$
\psi(\xi)=-i b \cdot \xi+\frac{1}{2} \xi \cdot Q \xi+\int_{y \neq 0}\left(1-e^{i y \cdot \xi}+i y \cdot \xi \mathbb{1}_{(0,1)}(|y|)\right) v(d y), \quad \xi \in \mathbb{R}^{d},
$$

where the Lévy triplet $(b, Q, v)$ consists of a vector $b \in \mathbb{R}^{d}$ (drift vector), a symmetric positive semi-definite matrix $Q \in \mathbb{R}^{d \times d}$ (diffusion matrix) and a measure $v$ on $\mathbb{R}^{d} \backslash\{0\}$ with $\int_{y \neq 0} \min \left\{1,|y|^{2}\right\} v(d y)<\infty$ (Lévy measure). Our standard references for Lévy processes are the monographs $[15,16,30]$. By the independence and stationarity of the increments, every Lévy process is a time-homogeneous Markov process, i.e. $P_{t} f(x):=\mathbb{E} f\left(x+X_{t}\right)$ defines a Markov semigroup on $\mathcal{B}_{b}\left(\mathbb{R}^{d}\right)$. We denote by $(A, \mathcal{D}(A))$ the weak infinitesimal generator,

$$
\begin{aligned}
& \mathcal{D}(A):=\left\{f \in \mathcal{B}_{b}\left(\mathbb{R}^{d}\right) ; \exists g \in \mathcal{B}_{b}\left(\mathbb{R}^{d}\right) \forall x \in \mathbb{R}^{d}: \lim _{t \rightarrow 0} \frac{\mathbb{E} f\left(x+X_{t}\right)-f(x)}{t}=g(x)\right\}, \\
& A f(x):=\lim _{t \rightarrow 0} \frac{\mathbb{E} f\left(x+X_{t}\right)-f(x)}{t}, \quad f \in \mathcal{D}(A) .
\end{aligned}
$$

If $f \in C_{b}^{2}\left(\mathbb{R}^{d}\right)$ then $f \in \mathcal{D}(A)$ and

$$
\begin{aligned}
A f(x)= & b \cdot \nabla f(x)+\frac{1}{2} \nabla \cdot Q \nabla f(x) \\
& +\int_{y \neq 0}\left(f(x+y)-f(x)-\nabla f(x) \cdot y \mathbb{1}_{(0,1)}(|y|)\right) v(d y) .
\end{aligned}
$$

Restricted to $C_{c}^{\infty}\left(\mathbb{R}^{d}\right)$, the infinitesimal generator is a pseudo-differential operator with symbol $\psi$,

$$
A f(x)=-\int_{\mathbb{R}^{d}} \psi(\xi) e^{i x \cdot \xi} \hat{f}(\xi) d \xi, \quad f \in C_{c}^{\infty}\left(\mathbb{R}^{d}\right)
$$

where $\hat{f}(\xi)=(2 \pi)^{-d} \int_{\mathbb{R}^{d}} f(x) e^{-i x \cdot \xi} d x$ is the Fourier transform of $f$. The pseudodifferential $A^{*}$ with symbol $\overline{\psi(\xi)}=\psi(-\xi)$ is the formal adjoint of $A$, in the sense that,

$$
\forall f, g \in C_{c}^{\infty}\left(\mathbb{R}^{d}\right): \int_{\mathbb{R}^{d}} A f(x) g(x) d x=\int_{\mathbb{R}^{d}} f(x) A^{*} g(x) d x .
$$

Given an open set $U \subseteq \mathbb{R}^{d}$ and $g \in L^{\infty}(U)$, a function $f$ is called a weak solution to $A f=g$ in $U$ if

$$
\forall \varphi \in C_{c}^{\infty}(U): \int_{\mathbb{R}^{d}} f(x) A^{*} \varphi(x) d x=\int_{U} g(x) \varphi(x) d x .
$$

It is implicitly assumed that the integral on the left-hand side exists; a sufficient condition is $f \in L^{\infty}\left(\mathbb{R}^{d}\right)$, see e.g. [6, Lemma 2.1] and [21, Proposition 2.1] for milder growth conditions on $f$. 


\section{Proofs}

In this section we present the proofs of our main results. Corollary 1 and 2 are consequences of Theorem 1, and therefore the main part is to establish Theorem 1. The idea is to combine the global Schauder estimates from [18] with a truncation technique to establish interior Schauder estimates. We start with the following auxiliary result.

Lemma 2 Let $\left(X_{t}\right)_{t \geq 0}$ be a Lévy process with weak infinitesimal generator $(A, \mathcal{D}(A))$ and Lévy triplet $(b, Q, v)$. If $f, g \in C_{b}^{2}\left(\mathbb{R}^{d}\right)$ then $f g \in \mathcal{D}(A)$ and

$$
A(f g)=g A f+f A g+\Gamma(f, g)
$$

where

$\Gamma(f, g)(x):=\nabla f(x) \cdot Q \nabla g(x)+\int_{y \neq 0}(f(x+y)-f(x))(g(x+y)-g(x)) v(d y), \quad x \in \mathbb{R}^{d}$,

is the Carré du Champ operator.

Proof Clearly, $f \cdot g \in C_{b}^{2}\left(\mathbb{R}^{d}\right) \subseteq \mathcal{D}(A)$. The identity for $A(f \cdot g)$ follows by applying (8) to $f \cdot g$ and rearranging the terms.

In contrast to other authors, we consider the Carré du champ operator $\Gamma$ as an operator on $C_{b}^{2}\left(\mathbb{R}^{d}\right)$ and not on $L^{2}(d x)$. For further information on the Carré du champ operator we refer the reader to $[2,3,13]$. Let us mention that the regularity assumptions in Lemma 2 can be relaxed. Roughly speaking, the identity holds whenever $f, g \in \mathcal{D}(A)$ are sufficiently smooth to make sense of $\Gamma(f, g)$; e.g. if $Q=0$ then $f, g$ need to satisfy a certain Hölder condition, see [18, Theorem 4.3] and [19].

The following a priori estimate is the core of the proof of our first main result, Theorem 1.

Proposition 1 Let $\left(X_{t}\right)_{t \geq 0}$ be a Lévy process with weak infinitesimal generator $(A, \mathcal{D}(A))$, characteristic exponent $\psi$ and Lévy triplet $(b, Q, v)$. If $(\mathrm{C} 1)-(\mathrm{C} 3)$ hold, then there exists for every $R>0$ and $\delta>0$ some constant $c>0$ such that

$$
\|f\|_{\mathcal{C}_{b}^{\alpha}(B(x, R))} \leq c\left(\|f\|_{\infty}+\|A f\|_{\infty, B(x, R+\delta)}\right)
$$

for all $f \in C_{b}^{2}\left(\mathbb{R}^{d}\right)$ and $x \in \mathbb{R}^{d}$; here $\alpha \in(0,2]$ denotes the constant from (C2).

In the proof of Proposition 1 we will use the elementary inequalities

$$
\begin{aligned}
\sup _{|h| \leq R} \frac{\left|\Delta_{h}^{3} f(x)\right|}{|h|^{\alpha}} & \leq 8 r^{-\alpha}\|f\|_{\infty}+\sup _{|h| \leq r} \frac{\left|\Delta_{h}^{3} f(x)\right|}{|h|^{\alpha}} \\
& \leq 8 r^{-\alpha}\|f\|_{\infty}+r^{\varepsilon} \sup _{|h| \leq r} \frac{\left|\Delta_{h}^{3} f(x)\right|}{|h|^{\alpha+\varepsilon}},
\end{aligned}
$$

which hold for any $0<r<R \leq 1, \varepsilon>0, f \in \mathcal{B}_{b}\left(\mathbb{R}^{d}\right)$ and $x \in \mathbb{R}^{d}$.

Proof of Proposition 1 For simplicity of notation we consider $x_{0}=0$ and $R=\delta=1$, i.e. we need to show

$$
\|f\|_{\mathcal{C}_{b}^{\alpha}(B(0,1))} \leq c\left(\|f\|_{\infty}+\|A f\|_{\infty, B(0,2)}\right) .
$$


Let $\chi \in C_{c}^{\infty}\left(\mathbb{R}^{d}\right)$ be such that $\mathbb{1}_{B(0,1 / 4)} \leq \chi \leq \mathbb{1}_{B(0,1 / 2)}$. For fixed $x \in B(0,2)$ define $r_{x}:=d\left(x, B(0,2)^{c}\right)$ and $\chi^{(x)}(y):=\chi\left(r_{x}^{-1}(y-x)\right)$. As $r_{x} \leq 2$, it follows from the chain rule that

$$
\left\|\chi^{(x)}\right\|_{C_{b}^{2}\left(\mathbb{R}^{d}\right)} \leq\left(1+r_{x}^{-2}\right)\|\chi\|_{C_{b}^{2}\left(\mathbb{R}^{d}\right)} \leq 5 r_{x}^{-2}\|\chi\|_{C_{b}^{2}\left(\mathbb{R}^{d}\right)} .
$$

We split the proof in several steps.

Step 1: There exist constants $\varepsilon \in(0, \alpha), \varrho>0$ and $C_{1}>0$ such that

$$
\left\|f \chi^{(x)}\right\|_{\mathcal{C}_{b}^{\alpha}\left(\mathbb{R}^{d}\right)} \leq C_{1}\left(r_{x}^{-\alpha-\varrho}\|f\|_{\infty}+\|A f\|_{\infty, B(0,2)}+\sup _{z \in B\left(x, 7 r_{x} / 8\right)} K(x, z)\right)
$$

for all $x \in B(0,2)$ and $f \in C_{b}^{2}\left(\mathbb{R}^{d}\right)$, where

$$
K(x, z):=\sup _{|h| \leq r_{x} / 32} \frac{\left|\Delta_{h}^{3} f(z)\right|}{|h|^{\alpha-\varepsilon}}
$$

Indeed We fix $x \in(0,2)$ and write $r:=r_{x}$ for brevity. Since $f \chi^{(x)}$ is twice continuously differentiable and vanishing at infinity, it is contained in the domain of the strong infinitesimal generator, and because of $(\mathrm{C} 1),(\mathrm{C} 2)$ we can apply [18, Theorem 1.1] to find that

$$
\left\|f \chi^{(x)}\right\|_{\mathcal{C}_{b}^{\alpha}\left(\mathbb{R}^{d}\right)} \leq c_{1}\left(\|f\|_{\infty}+\left\|A\left(f \chi^{(x)}\right)\right\|_{\infty}\right)
$$

for some constant $c_{1}>0$ which does not depend on $f$ and $x$. Hence, by Lemma 2 ,

$$
\left\|f \chi^{(x)}\right\|_{\mathcal{C}_{b}^{\alpha}\left(\mathbb{R}^{d}\right)} \leq c_{1}\left(\|f\|_{\infty}+\left\|\chi^{(x)} A f\right\|_{\infty}+\left\|f A \chi^{(x)}\right\|_{\infty}+\left\|\Gamma\left(f, \chi^{(x)}\right)\right\|_{\infty}\right) .
$$

As $0 \leq \chi^{(x)} \leq 1, \operatorname{supp} \chi^{(x)} \subseteq B(0,2)$ and

$$
\left\|A \chi^{(x)}\right\|_{\infty} \leq c_{2}\left\|\chi^{(x)}\right\|_{C_{b}^{2}\left(\mathbb{R}^{d}\right)} \leq 5 c_{2} r^{-2}\|\chi\|_{C_{b}^{2}\left(\mathbb{R}^{d}\right)},
$$

it holds that

$$
\left\|f \chi^{(x)}\right\|_{\mathcal{C}_{b}^{\alpha}\left(\mathbb{R}^{d}\right)} \leq\left(1+5 c_{2}\right)\left(r^{-2}\|f\|_{\infty}+\|A f\|_{\infty, B(0,2)}+\left\|\Gamma\left(f, \chi^{(x)}\right)\right\|_{\infty}\right) .
$$

It remains to estimate the supremum norm of the term involving the Carré du champ operator.

$\mathbf{1}^{\mathrm{o}}$ For $z \in \mathbb{R}^{d} \backslash B(x, 3 r / 4)$ we have $\chi^{(x)}=0$ on $B(z, r / 4)$, and therefore

$$
\Gamma\left(f, \chi^{(x)}\right)(z)=\int_{|y|>r / 4}(f(z+y)-f(z))\left(\chi^{(x)}(z+y)-\chi^{(x)}(z)\right) v(d y)
$$

implying

$$
\begin{aligned}
\left|\Gamma\left(f, \chi^{(x)}\right)(z)\right| & \leq 4\|f\|_{\infty} \int_{|y|>r / 4} v(d y) \\
& \leq 4^{1+\gamma} r^{-\gamma}\|f\|_{\infty} \int_{y \neq 0} \min \left\{1,|y|^{\gamma}\right\} v(d y) .
\end{aligned}
$$

Since $\int_{y \neq 0} \min \left\{1,|y|^{\gamma}\right\} \vee(d y)<\infty$, cf. (C3), and $r \leq 2$, this implies

$$
\left|\Gamma\left(f, \chi^{(x)}\right)(z)\right| \leq c_{3} r^{-\alpha-\varrho}\|f\|_{\infty}, \quad z \in \mathbb{R}^{d} \backslash B(x, 3 r / 4),
$$

for suitable constants $c_{3}>0$ and $\varrho>0$, which is an estimate of the desired form. 
$2^{\mathrm{O}}$ For $z \in B(x, 3 r / 4)$ we consider the local term and the non-local term separately, i.e. we write $\Gamma\left(f, \chi^{(x)}\right)(z)=D(z)+I(z)$ where

$$
D(z):=\nabla f(z) \cdot Q \nabla \chi^{(x)}(z)
$$

and

$$
I(z):=\int_{y \neq 0}(f(z+y)-f(z))\left(\chi^{(x)}(z+y)-\chi^{(x)}(z)\right) v(d y) .
$$

For the local term it clearly suffices to consider the case $Q \neq 0$. If $Q \neq 0$ then, by (C3), $\alpha>1$ and therefore we can choose $\varepsilon \in(0,1)$ such that $\alpha-2 \varepsilon>1$. Clearly,

$$
|D(z)| \leq\left\|\chi^{(x)}\right\|_{C_{b}^{1}\left(\mathbb{R}^{d}\right)}\|Q\||\nabla f(z)| \leq 3 r^{-1}\|Q\|\|\chi\|_{C_{b}^{1}\left(\mathbb{R}^{d}\right)}|\nabla f(z)| .
$$

Since there exists a constant $c_{4}>0$ such that

$$
|\nabla f(z)| \leq c_{4} r^{-\alpha}\|f\|_{\infty}+c_{4} \sup _{|y-z| \leq r / 16} \sup _{|h| \leq 1} \frac{\left|\Delta_{h}^{3} f(y)\right|}{|h|^{\alpha-2 \varepsilon}},
$$

cf. Lemma 1.ii), it follows from Eqs. 10 and 11 that

$$
\begin{aligned}
|\nabla f(z)| \leq & c_{4}^{\prime} r^{-2 \theta-\alpha}\|f\|_{\infty} \\
& +c_{4}^{\prime} \sup \left\{\frac{\left|\Delta_{h}^{3} f(y)\right|}{|h|^{\alpha-2 \varepsilon}} ; y \in B(x, 7 r / 8),|h| \leq \frac{1}{32}\left(\frac{r}{2}\right)^{\theta}\right\} \\
\leq & c_{4}^{\prime} r^{-2 \theta-\alpha}\|f\|_{\infty} \\
& +c_{4}^{\prime \prime} r^{\theta \varepsilon} \sup \left\{\frac{\left|\Delta_{h}^{3} f(y)\right|}{|h|^{\alpha-\varepsilon}} ; y \in B(x, 7 r / 8),|h| \leq \frac{1}{32}\left(\frac{r}{2}\right)^{\theta}\right\}
\end{aligned}
$$

for any $\theta>0$ and $z \in B(x, 3 r / 4)$. Choosing $\theta:=1 / \varepsilon$ we get

$$
\begin{aligned}
|D(z)| \leq & c_{5} r^{-1-2 / \varepsilon-\alpha}\|f\|_{\infty} \\
& +c_{5} \sup \left\{\frac{\left|\Delta_{h}^{3} f(y)\right|}{|h|^{\alpha-\varepsilon}} ; y \in B(x, 7 r / 8),|h| \leq \frac{1}{32}\left(\frac{r}{2}\right)^{\theta}\right\}
\end{aligned}
$$

for all $z \in B(x, 3 r / 4)$. As $r / 2 \leq 1$ and $\theta>1$, the supremum over $|h| \leq \frac{1}{32}(r / 2)^{\theta}$ is less or equal than the supremum over $|h| \leq \frac{1}{32}(r / 2)$, and so

$$
|D(z)| \leq c_{5} r^{-1-2 / \varepsilon-\alpha}\|f\|_{\infty}+c_{5} \sup _{y \in B(x, 7 r / 8)} K(x, y)
$$

with $K$ defined in Eq. 13. It remains to estimate the non-local term. We consider the cases $\gamma=2$ and $\gamma \in(0,2)$ separately. If $\gamma=2$, then by (C3) $\alpha>1$. Choose $\varepsilon \in(0,1)$ such that $\alpha-2 \varepsilon>1$ and set $\theta=1 / \varepsilon$. By the Lipschitz continuity of $\chi^{(x)}$,

$$
\begin{aligned}
|I(z)| \leq & \left\|\chi^{(x)}\right\|_{C_{b}^{1}\left(\mathbb{R}^{d}\right)} \int_{|y| \leq r / 32}|f(y+z)-f(z)||y| v(d y) \\
& +4\|f\|_{\infty} \int_{|y|>r / 32} v(d y) .
\end{aligned}
$$

Since $\int_{y \neq 0} \min \left\{1,|y|^{\gamma}\right\} v(d y)<\infty$ and $\left\|\chi^{(x)}\right\|_{C_{b}^{1}\left(\mathbb{R}^{d}\right)} \leq 3 r^{-1}\|\chi\|_{C_{b}^{1}\left(\mathbb{R}^{d}\right)}$, this implies that there exists a finite constant $c_{6}>0$ such that

$$
|I(z)| \leq c_{6} r^{-1}\|\chi\|_{C_{b}^{1}\left(\mathbb{R}^{d}\right)} \sup _{|h| \leq r / 32}|\nabla f(z+h)| \int_{|y| \leq r / 32}|y|^{2} v(d y)+c_{6} r^{-\gamma}\|f\|_{\infty} .
$$


For the first term on the right-hand side we can now use a reasoning similar to that for the local term and get the required estimate.

Finally we consider the case $\gamma<2$. Fix $\varepsilon \in(0,1)$ such that $\max \{0, \gamma-1\}<\alpha-2 \varepsilon-$ it exists because of $(\mathrm{C} 3)-$ and set $\theta:=1 / \varepsilon$. We have

$$
\begin{aligned}
|I(z)| \leq & \left\|\chi^{(x)}\right\|_{C_{b}^{1}\left(\mathbb{R}^{d}\right)} \sup _{|h| \leq 1} \frac{|f(z+h)-f(z)|}{|h|^{\max \{0, \gamma-1\}}} \int_{|y| \leq 1}|y|^{\gamma} v(d y) \\
& +4\|f\|_{\infty} \int_{|y|>1} v(d y) .
\end{aligned}
$$

Using that $\int \min \left\{1,|y|^{\gamma}\right\} v(d y)<\infty$ and $\left\|\chi^{(x)}\right\|_{C_{b}^{1}\left(\mathbb{R}^{d}\right)} \leq 3 r^{-1}\|\chi\|_{C_{b}^{1}\left(\mathbb{R}^{d}\right)}$, we find a finite constant $c_{7}>0$ such that

$$
|I(z)| \leq c_{7}\|f\|_{\infty}+c_{7} r^{-1} \sup _{|h| \leq 1} \frac{|f(z+h)-f(z)|}{|h|^{\max \{0, \gamma-1\}}} .
$$

Since $\max \{0, \gamma-1\}<1$, an application of Lemma 1.i) shows that

$$
\sup _{|h| \leq 1} \frac{|f(z+h)-f(z)|}{|h|^{\max \{0, \gamma-1\}}} \leq c_{8} r^{-1}\|f\|_{\infty}+c_{8} \sup _{|h| \leq 1} \sup _{|y-z| \leq r / 16} \frac{\left|\Delta_{h}^{3} f(y)\right|}{|h|^{\max \{0, \gamma-1\}}} .
$$

Hence, by Eqs. 10 and 11,

$$
\begin{aligned}
& \sup _{|y| \leq 1} \frac{|f(z+y)-f(z)|}{|y|^{\max \{0, \gamma-1\}}} \\
& \leq c_{8}^{\prime} r^{-\theta}\|f\|_{\infty}+c_{8} r^{\theta \varepsilon} \sup \left\{\frac{\left|\Delta_{h}^{3} f(y)\right|}{|h|^{\alpha-\varepsilon}} ; y \in B(x, 7 r / 8),|h| \leq \frac{1}{32}\left(\frac{r}{2}\right)^{\theta}\right\}
\end{aligned}
$$

for all $z \in B(x, 3 r / 4)$. Recalling that $\theta \varepsilon=1$ and $r \leq 2$ we conclude that

$$
\begin{aligned}
|I(z)| & \leq c_{9} r^{-\theta-1}\|f\|_{\infty}+c_{9} \sup \left\{\frac{\left|\Delta_{h}^{3} f(y)\right|}{|h|^{\alpha-\varepsilon}} ; y \in B(x, 7 r / 8),|h| \leq \frac{1}{32}\left(\frac{r}{2}\right)^{\theta}\right\} \\
& \leq c_{10} r^{-\alpha-\varrho}\|f\|_{\infty}+c_{9} \sup _{y \in B(x, 7 r / 8)} K(x, y)
\end{aligned}
$$

where $\varrho:=\max \{1, \theta+1-\alpha\}$.

Step 2: There exists a constant $C_{2}>0$ such that

$$
|f|_{B(0,2), \alpha, \varrho} \leq C_{2}\left(\|f\|_{\infty}+\|A f\|_{\infty, B(0,2)}+|f|_{B(0,2), \alpha-\varepsilon, \varrho}\right),
$$

where $\varrho, \varepsilon$ are the constants from Step 1 and

$$
|f|_{B(0,2), \sigma, \kappa}:=\sup _{x \in B(0,2)} \sup _{|h| \leq r_{x} / 4} r_{x}^{\kappa+\sigma} \frac{\left|\Delta_{h}^{3} f(x)\right|}{|h|^{\sigma}}, \quad \kappa>0, \sigma \in(0,3) .
$$

Indeed For $z \in B\left(x, 7 r_{x} / 8\right)$ and $x \in B(0,2)$ we have $r_{z} \geq r_{x} / 8$, and therefore the mapping $K$ defined in Eq. 13 satisfies

$$
\begin{aligned}
r_{x}^{\alpha+\varrho} \sup _{z \in B\left(x, 7 r_{x} / 8\right)} K(x, z) & \leq 8^{\alpha+\varrho} \sup _{z \in B\left(x, 7 r_{x} / 8\right)} \sup _{|h| \leq r_{x} / 32} r_{z}^{\alpha+\varrho} \frac{\left|\Delta_{h}^{3} f(z)\right|}{|h|^{\alpha-\varepsilon}} \\
& \leq 8^{\alpha+\varrho} \sup _{z \in B(0,2)} \sup _{|h| \leq r_{z} / 4} r_{z}^{\alpha+\varrho} \frac{\left|\Delta_{h}^{3} f(z)\right|}{|h|^{\alpha-\varepsilon}} \\
& \leq 28^{\alpha+\varrho}|f|_{B(0,2), \alpha-\varepsilon, \varrho .}
\end{aligned}
$$


Consequently, it follows from Step 1 that

$$
r_{x}^{\varrho+\alpha}\left\|f \chi^{(x)}\right\|_{\mathcal{C}_{b}^{\alpha}\left(\mathbb{R}^{d}\right)} \leq C_{1}\left(\|f\|_{\infty}+\|A f\|_{\infty, B(0,2)}+28^{\alpha+\varrho}|f|_{B(0,2), \alpha-\varepsilon, \varrho}\right)
$$

for all $x \in B(0,2)$. As $\alpha \in(0,2]$, this gives

$$
r_{x}^{\varrho+\alpha} \sup _{|h| \leq 1} \sup _{z \in \mathbb{R}^{d}} \frac{\left|\Delta_{h}^{3}\left(f \chi^{(x)}\right)(z)\right|}{|h|^{\alpha}} \leq c_{2}\left(\|f\|_{\infty}+\|A f\|_{\infty, B(0,2)}+|f|_{B(0,2), \alpha-\varepsilon, \varrho}\right)
$$

for all $x \in B(0,2)$ and some constant $c_{2}>0$ not depending on $x$ and $f$, cf. Eq. 7. Since $\chi^{(x)}=1$ on $B\left(x, r_{x} / 4\right)$, this implies

$$
r_{x}^{\varrho+\alpha} \sup _{|h| \leq r_{x} / 12} \frac{\left|\Delta_{h}^{3} f(x)\right|}{|h|^{\alpha}} \leq c_{2}\left(\|f\|_{\infty}+\|A f\|_{\infty, B(0,2)}+|f|_{B(0,2), \alpha-\varepsilon, \varrho}\right) .
$$

On the other hand, we have

$$
r_{x}^{\varrho+\alpha} \sup _{r_{x} / 12 \leq|h| \leq r_{x} / 4} \frac{\left|\Delta_{h}^{3} f(x)\right|}{|h|^{\alpha}} \leq 8 r_{x}^{\varrho+\alpha}\|f\|_{\infty}\left(\frac{r_{x}}{12}\right)^{-\alpha} \leq c_{3}\|f\|_{\infty}
$$

for some uniform constant $c_{3}>0$. Combining both estimates yields

$$
r_{x}^{\varrho+\alpha} \sup _{|h| \leq r_{x} / 4} \frac{\left|\Delta_{h}^{3} f(x)\right|}{|h|^{\alpha}} \leq c_{4}\left(\|f\|_{\infty}+\|A f\|_{\infty, B(0,2)}+|f|_{B(0,2), \alpha-\varepsilon, \varrho}\right)
$$

for all $x \in B(0,2)$, and this proves (16).

Step 3: Choose $\delta \in\left(0, \frac{1}{4}\right)$ sufficiently small such that $C_{2} \delta \leq \frac{1}{2}$ for the constant $C_{2}$ from Step 2. By definition, cf. Eq. 17,

$$
|f|_{B(0,2), \alpha-\varepsilon, \varrho}=\sup _{x \in B(0,2) h:\left|h / r_{x}\right| \leq 1 / 4} r_{x}^{\varrho}\left(\frac{r_{x}}{|h|}\right)^{\alpha-\varepsilon}\left|\Delta_{h}^{3} f(x)\right| .
$$

Since

$$
\begin{aligned}
\sup _{h:\left|h / r_{x}\right| \leq \delta^{1 / \varepsilon}} r_{x}^{\varrho}\left(\frac{r_{x}}{|h|}\right)^{\alpha-\varepsilon}\left|\Delta_{h}^{3} f(x)\right| & \leq \delta \sup _{h:\left|h / r_{x}\right| \leq \delta^{1 / \varepsilon}} r_{x}^{\varrho}\left(\frac{r_{x}}{|h|}\right)^{\alpha}\left|\Delta_{h}^{3} f(x)\right| \\
& \leq \delta|f|_{B(0,2), \alpha, \varrho}
\end{aligned}
$$

and

$$
\begin{aligned}
\sup _{h: \delta^{1 / \varepsilon}<\left|h / r_{x}\right| \leq 1 / 4} r_{x}^{\varrho}\left(\frac{r_{x}}{|h|}\right)^{\alpha-\varepsilon}\left|\Delta_{h}^{3} f(x)\right| & \leq \delta^{-\alpha / \varepsilon} \sup _{h: \delta^{1 / \varepsilon}<\left|h / r_{x}\right| \leq 1 / 4} r_{x}^{\varrho}\left|\Delta_{h}^{3} f(x)\right| \\
& \leq 8 \delta^{-\alpha / \varepsilon} 2^{\varrho}\|f\|_{\infty},
\end{aligned}
$$

it follows that there exists a constant $c_{1}>0$ such that

$$
|f|_{B(0,2), \alpha-\varepsilon, \varrho} \leq \delta|f|_{B(0,2), \alpha, \varrho}+c_{1}\|f\|_{\infty} .
$$

As $C_{2} \delta \leq \frac{1}{2}$, we find from Step 2 and Eq. 19 that

$$
\begin{aligned}
|f|_{B(0,2), \alpha, \varrho} & \leq C_{2}\left(\|f\|_{\infty}+\|A f\|_{\infty, B(0,2)}+|f|_{B(0,2), \alpha-\varepsilon, \varrho}\right) \\
& \leq C_{2}\left(\left(1+c_{1}\right)\|f\|_{\infty}+\|A f\|_{\infty, B(0,2)}\right)+\frac{1}{2}|f|_{B(0,2), \alpha, \varrho},
\end{aligned}
$$

i.e.

$$
|f|_{B(0,2), \alpha, \varrho} \leq c_{2}\left(\|f\|_{\infty}+\|A f\|_{\infty, B(0,2)}\right) .
$$


On the other hand, we also have

$$
|f|_{B(0,2), \alpha, \varrho} \geq \sup _{x \in B(0,1)} \sup _{|h| \leq 1 / 4} \frac{\left|\Delta_{h}^{3} f(x)\right|}{|h|^{\alpha}} .
$$

Combining both estimates and applying Lemma 1, the assertion follows.

The seminorms $|f|_{U, \alpha, \varrho}$ which we introduced in Eq. 17 are closely related to seminorms which appear in the study of Schauder estimates for second order differential operators, cf. [8]; our definition is inspired by Hölder-Zygmund norms whereas the seminorms in [8] are based on "standard" Hölder norms.

In order to apply our a priori estimate from Proposition 1, we have to approximate the weak solution $f$ by a sequence $\left(f_{k}\right)_{k \geq 1}$ of twice differentiable functions; it is a natural idea to consider $f_{k}:=f * \chi_{k}$ for a suitable sequence of mollifiers $\left(\chi_{k}\right)_{k \geq 1}$. To make this approximation work, we need to know that $A f_{k}$ is on $U$ close to $A f=g$, and this is what the next lemma is about.

Lemma 3 Let $\left(X_{t}\right)_{t \geq 0}$ be a Lévy process with weak infinitesimal generator $(A, \mathcal{D}(A))$. Let $f \in L^{\infty}\left(\mathbb{R}^{d}\right)$ be a weak solution to the equation $A f=g$ in $U$ for $g \in L^{\infty}(U)$ and $U \subseteq \mathbb{R}^{d}$ open. If $\chi \in C_{c}^{\infty}(B(0, r))$ for some $r>0$, then $f * \chi \in \mathcal{D}(A)$ and $A(f * \chi)(x)=(g * \chi)(x)$ for all $x \in U$ with $B(x, r) \subseteq U$.

Proof It is well known that $u:=f * \chi \in C_{b}^{\infty}\left(\mathbb{R}^{d}\right)$ and

$$
\partial^{\alpha} u=f *\left(\partial^{\alpha} \chi\right), \quad \alpha \in \mathbb{N}_{0}^{d},
$$

see e.g. [35]. In particular, $u \in C_{b}^{2}\left(\mathbb{R}^{d}\right) \subseteq \mathcal{D}(A)$ and

$$
A u(x)=b \cdot \nabla u(x)+\frac{1}{2} \nabla \cdot Q \cdot \nabla u(x)+\int_{y \neq 0}\left(u(x+y)-u(x)-\nabla u(x) \cdot y \mathbb{1}_{(0,1)}(|y|)\right) v(d y) .
$$

Using Eq. 20 and Fubini's theorem it follows that

$$
A u(x)=(f * A \chi)(x)=\int_{\mathbb{R}^{d}} f(y)(A \chi)(x-y) d y
$$

for all $x>\mathbb{R}^{d}$.

$$
\left(A^{\chi}\right)(x-y)=\left(A *_{\chi}(x-\cdot)\right)(y)
$$

for the formal adjoint $A^{*}$, we get

$$
A u(x)=\int_{\mathbb{R}^{d}} f(y)\left(A^{*} \chi(x-\cdot)\right)(y) d y .
$$

If $x \in U$ is such that $B(x, r) \subseteq U$, then supp $\chi(x-\cdot) \subseteq U$ and so it follows from the definition of the weak solution, cf. Eq. 9 , that

$$
A u(x)=\int_{\mathbb{R}^{d}} g(y) \chi(x-y) d y=(g * \chi)(x)
$$

for any $\operatorname{such} x$.

We are now ready to prove Theorem 1 . 
Proof of Theorem 1 Let $f \in L^{\infty}\left(\mathbb{R}^{d}\right)$ and $g \in L^{\infty}(U)$ be such that $A f=g$ weakly in $U$. Pick $\chi \in C_{c}^{\infty}\left(\mathbb{R}^{d}\right)$ such that $\chi \geq 0$, supp $\chi \subseteq B(0,1), \int \chi(x) d x=1$ and set $\chi_{k}(x):=k^{d} \chi(k x)$. If we define

$$
f_{k}(x):=\left(f * \chi_{k}\right)(x):=\int_{\mathbb{R}^{d}} f(y) \chi_{k}(x-y) d y,
$$

then Lemma 3 shows that $f_{k} \in C_{b}^{2}\left(\mathbb{R}^{d}\right) \subseteq \mathcal{D}(A)$ and

$$
A f_{k}(x)=g_{k}(x):=\left(g * \chi_{k}\right)(x) \text { for all } x \in U \text { with } B(x, 1 / k) \subseteq U .
$$

i). Set $U_{\delta}:=\left\{x \in U ; d\left(x, U^{c}\right)>\delta\right\}$ for $\delta>0$. By Proposition 1, there exists a constant $c_{1}=c_{1}(\delta)$ such that

$$
\left\|f_{k}\right\|_{\mathcal{C}_{b}^{\alpha}(B(x, \delta / 2))} \leq c_{1}\left(\left\|f_{k}\right\|_{\infty}+\left\|A f_{k}\right\|_{\infty, U_{\delta / 4}}\right)
$$

for all $x \in U_{\delta}$ and $k \geq 1$. Choosing $k \gg 1$ sufficiently large such that $\frac{1}{k}<\frac{\delta}{4}$ we find from Eq. 21 and $\left\|f_{k}\right\|_{\infty} \leq\|f\|_{L^{\infty}\left(\mathbb{R}^{d}\right)}$ that

$$
\left\|f_{k}\right\|_{\mathcal{C}_{b}^{\alpha}(B(x, \delta / 2))} \leq c_{1}\left(\|f\|_{L^{\infty}\left(\mathbb{R}^{d}\right)}+\|g\|_{L^{\infty}(U)}\right) \quad \text { for all } x \in U_{\delta} .
$$

By the Arzelà-Ascoli theorem, there exists a continuous function $f^{(\delta)}: U_{\delta} \rightarrow \mathbb{R}$ such that a subsequence $f_{k_{j}}$ converges pointwise to $f^{(\delta)}$ on $U_{\delta}$. By Eq. 22, we have

$$
\frac{\left|\Delta_{h}^{N} f^{(\delta)}(x)\right|}{|h|^{\alpha}}=\lim _{j \rightarrow \infty} \frac{\left|\Delta_{h}^{N} f_{k_{j}}(x)\right|}{|h|^{\alpha}} \leq c_{1}\left(\|f\|_{L^{\infty}\left(\mathbb{R}^{d}\right)}+\|g\|_{L^{\infty}(U)}\right)
$$

for all $x \in U_{2 \delta}$ and $|h| \leq \delta / 3$ where $N=\lfloor\alpha\rfloor+1 \in\{1,2,3\}$ is the smallest integer larger than $\alpha$. Hence,

$$
\left\|f^{(\delta)}\right\| \mathcal{C}_{b}^{\alpha}\left(U_{2 \delta}\right) \leq c_{2}\left(\|f\|_{L^{\infty}\left(\mathbb{R}^{d}\right)}+\|g\|_{L^{\infty}(U)}\right) .
$$

On the other hand, $f_{k} \rightarrow f$ in $L^{1}(d x)$ and so $f=f^{(\delta)}$ Lebesgue almost everywhere on $U_{\delta}$. As $f^{\left(\delta^{\prime}\right)}=f^{(\delta)}$ on $U_{\delta}$ for $\delta^{\prime}<\delta$, the mapping

$$
\tilde{f}(x):= \begin{cases}f^{(\delta)}(x), & \text { if } x \in U_{\delta} \text { for some } \delta>0, \\ f(x), & \text { if } x \in U^{c},\end{cases}
$$

is well defined. Clearly, $\tilde{f}=f$ Lebesgue almost everywhere and the interior Schauder estimate

$$
\|\tilde{f}\|_{\mathcal{C}_{b}^{\alpha}\left(U_{\delta}\right)} \leq C_{\delta}\left(\|f\|_{L^{\infty}\left(\mathbb{R}^{d}\right)}+\|g\|_{L^{\infty}(U)}\right)
$$

holds for all $\delta>0$.

ii). Assume now additionally that $f \in \mathcal{C}_{b}^{\kappa}\left(\mathbb{R}^{d}\right)$ and $g \in \mathcal{C}_{b}^{\kappa}(U)$ for some $\kappa>0$. Since $\left.A\right|_{C_{b}^{2}\left(\mathbb{R}^{d}\right)}$ commutes with the shift operator, i.e.

$$
(A \varphi)\left(x+x_{0}\right)=\left(A \varphi\left(\cdot+x_{0}\right)\right)(x), \quad \varphi \in C_{b}^{2}\left(\mathbb{R}^{d}\right), x, x_{0} \in \mathbb{R}^{d},
$$

cf. Eq. 8, it follows easily by induction that

$$
A\left(\Delta_{h}^{N} \varphi\right)=\Delta_{h}^{N}(A \varphi) \quad \text { for all } \varphi \in C_{b}^{2}\left(\mathbb{R}^{d}\right), N \in \mathbb{N} .
$$

Hence, by Eq. 21,

$$
A\left(\Delta_{h}^{N} f_{k}\right)(x)=\left(\Delta_{h}^{N} A f_{k}\right)(x)=\Delta_{h}^{N} g_{k}(x)
$$


for all $N \in \mathbb{N}, x \in U_{\delta},|h|<\delta /(2 N)$ and $k \gg 1$ with $\frac{1}{k}<\frac{\delta}{2}$. Choose $N=\lfloor\kappa\rfloor+1$. Applying part i) to $x \mapsto \Delta_{h}^{N} f_{k}(x)$, we obtain from Eq. 23 that

$$
\left\|\Delta_{h}^{N} f_{k}\right\|_{\mathcal{e}_{b}^{\alpha}\left(U_{2 \delta}\right)} \leq c_{1}\left(\left\|\Delta_{h}^{N} f_{k}\right\|_{\infty}+\left\|\Delta_{h}^{N} g_{k}\right\|_{\infty, U_{\delta}}\right)
$$

for every $\delta \in(0,1)$ and some constant $c_{1}=c_{1}(\delta)>0$ not depending on $f, g$ and $k$. Since $f \in C_{b}^{\kappa}\left(\mathbb{R}^{d}\right)$ and $g \in \mathcal{C}_{b}^{\kappa}(U)$, this implies

$$
\left\|\Delta_{h}^{N} f_{k}\right\| \mathcal{C}_{b}^{\alpha}\left(U_{2 \delta}\right) \leq c_{2}|h|^{\kappa}\left(\|f\|_{\mathcal{C}_{b}^{\kappa}\left(\mathbb{R}^{d}\right)}+\|g\|_{\mathcal{C}_{b}^{\kappa}(U)}\right)
$$

for all $|h|<\delta /(2 N)$. Set $M=\lfloor\alpha\rfloor+1$, then

$$
\left|\Delta_{h}^{M} \Delta_{h}^{N} f_{k}(x)\right| \leq c_{3}|h|^{\kappa+\alpha}\left(\|f\|_{\mathcal{C}_{b}^{\kappa}\left(\mathbb{R}^{d}\right)}+\|g\|_{\mathcal{C}_{b}^{\kappa}(U)}\right)
$$

for all $x \in U_{3 \delta}$ and small $h$. The left-hand side converges to $\Delta_{h}^{M} \Delta_{h}^{N} f(x)=$ $\Delta_{h}^{M+N} f(x)$ as $k \rightarrow \infty$ because $f$ is continuous. Applying Lemma 1 finishes the proof.

For the proof of Corollary 1 we need another auxiliary result.

Lemma 4 Let $\left(X_{t}\right)_{t \geq 0}$ be a Lévy process with characteristic exponent $\psi$ satisfying the Hartman-Wintner condition $(\mathrm{C} 1)$. Let $f \in \mathcal{B}_{b}\left(\mathbb{R}^{d}\right)$ be such that

$$
\lim _{t \rightarrow 0}\left|\mathbb{E} f\left(x+X_{t}\right)-f(x)\right|=0, \quad x \in U,
$$

for an open set $U \subseteq \mathbb{R}^{d}$. If $\tilde{f} \in C_{b}(U)$ is such that $f=\tilde{f}$ Lebesgue almost everywhere on $U$, then $f=\tilde{f}$ on $U$; in particular, $\left.f\right|_{U}$ is continuous.

Proof The function

$$
u:=\tilde{f} \mathbb{1}_{U}+f \mathbb{1}_{U^{c}},
$$

satisfies $u \in \mathcal{B}_{b}\left(\mathbb{R}^{d}\right)$ and $f=u$ Lebesgue almost everywhere on $\mathbb{R}^{d}$. Since the characteristic exponent $\psi$ satisfies the Hartman-Wintner condition, the law of $X_{t}$ has a density $p_{t}$ with respect to Lebesgue measure for $t>0$, and so

$$
\mathbb{E} u\left(x+X_{t}\right)=\mathbb{E} f\left(x+X_{t}\right) \quad \text { for all } x \in \mathbb{R}^{d}, t>0 .
$$

If we can show that

$$
\lim _{t \rightarrow 0} \mathbb{E} u\left(x+X_{t}\right)=u(x)=\tilde{f}(x) \quad \text { for all } x \in U,
$$

then it follows immediately from Eq. 24 that

$$
f(x)=\lim _{t \rightarrow 0} \mathbb{E} f\left(x+X_{t}\right)=\lim _{t \rightarrow 0} \mathbb{E} u\left(x+X_{t}\right)=\tilde{f}(x) \quad \text { for all } x \in U,
$$

which proves the assertion. To prove (25), fix $x \in U$. As

$$
\left|\mathbb{E} u\left(x+X_{t}\right)-u(x)\right| \leq \sup _{|y| \leq \delta}|u(x+y)-u(x)|+2\|u\|_{L^{\infty}\left(\mathbb{R}^{d}\right)} \mathbb{P}\left(\sup _{s \leq t}\left|X_{s}\right|>\delta\right),
$$

we find from the right-continuity of the sample paths and the monotone convergence theorem that

$$
\limsup _{t \rightarrow 0}\left|\mathbb{E} u\left(x+X_{t}\right)-u(x)\right| \leq \sup _{|y| \leq \delta}|u(x+y)-u(x)| .
$$

Since $u$ is continuous at $x$, the right-hand side tends to 0 as $\delta \rightarrow 0$, and this gives (25). 
Remark 2 The proof of Lemma 4 shows the following statement: If $\left(X_{t}\right)_{t \geq 0}$ is a Lévy process, then

$$
\lim _{t \rightarrow 0} \mathbb{E} f\left(x+X_{t}\right)=f(x)
$$

holds for any continuity point $x$ of $f \in \mathcal{B}_{b}\left(\mathbb{R}^{d}\right)$; this is a localized version of the continuity of the semigroup $T_{t} f(x):=\mathbb{E} f\left(x+X_{t}\right)$ at $t=0$.

Proof of Corollary 1 Let $\varphi \in C_{c}^{\infty}(U)$. Because of the compact uniform boundedness assumption (6), it follows from the dominated convergence theorem that

$$
\int_{\mathbb{R}^{d}} g(x) \varphi(x) d x=\lim _{t \rightarrow 0} \frac{1}{t}\left(\mathbb{E} \int_{\mathbb{R}^{d}} f\left(x+X_{t}\right) \varphi(x) d x-\int_{\mathbb{R}^{d}} f(x) \varphi(x) d x\right) .
$$

Performing a change of variables we get

$$
\begin{aligned}
\int_{\mathbb{R}^{d}} g(x) \varphi(x) d x & =\lim _{t \rightarrow 0} \frac{1}{t}\left(\mathbb{E} \int_{\mathbb{R}^{d}} f(x) \varphi\left(x-X_{t}\right) d x-\int_{\mathbb{R}^{d}} f(x) \varphi(x) d x\right) \\
& =\int_{\mathbb{R}^{d}} f(x) A^{*} \varphi(x) d x,
\end{aligned}
$$

i.e. $f$ is a weak solution to $A f=g$ on $U$. If $g \in L^{\infty}(U)$ then it follows from Theorem 1.i) that there exists a function $\tilde{f} \in L^{\infty}\left(\mathbb{R}^{d}\right)$ such that $f=\tilde{f}$ Lebesgue almost everywhere, $\tilde{f} \in C(U)$ and

$$
\|\tilde{f}\|_{\mathcal{C}_{b}^{\alpha}\left(U_{\delta}\right)} \leq C_{\delta}\left(\|f\|_{L^{\infty}\left(\mathbb{R}^{d}\right)}+\|g\|_{\infty, U}\right), \quad \delta>0 .
$$

By Lemma 4, we have $f=\tilde{f}$ on $U$, and consequently (26) holds with $\tilde{f}$ replaced by $f$; this proves the first assertion. The second assertion follows directly from Theorem 1.ii).

Proof of Corollary 2 The first assertion is immediate from Corollary 1.i). For ii), we note that $A f \in \mathcal{C}_{b}^{\kappa}\left(\mathbb{R}^{d}\right)$ implies $f \in \mathcal{C}_{b}^{\kappa+\alpha}\left(\mathbb{R}^{d}\right)$ and

$$
\|f\|_{\mathcal{C}_{b}^{\kappa}\left(\mathbb{R}^{d}\right)} \leq\|f\|_{\mathcal{C}_{b}^{\kappa+\alpha}\left(\mathbb{R}^{d}\right)} \leq c\left(\|f\|_{\infty}+\|A f\|_{\mathcal{C}_{b}^{\kappa}\left(\mathbb{R}^{d}\right)}\right),
$$

cf. [18, Theorem 1.1], and so Corollary 1.ii) applies.

\section{Examples}

In this section, we present examples of Lévy processes for which interior Schauder estimates can be obtained from Theorem 1, Corollary 1 and Corollary 2 . We start with two tools which are useful to construct wide classes of Lévy processes satisfying the assumptions (C1)-(C3) of our main results.

Proposition 2 Let $\left(X_{t}^{(i)}\right)_{t \geq 0}, i=1,2$, be independent $\mathbb{R}^{d_{i}}$-valued Lévy processes.

i). $\left(d_{1}=d_{2}\right)$ If $\left(X_{t}^{(1)}\right)_{t \geq 0}$ satisfies $(\mathrm{C} 1)$ and $(\mathrm{C} 2)$ for some $\alpha>0$, then the Lévy process

$$
Y_{t}:=X_{t}^{(1)}+X_{t}^{(2)}, \quad t \geq 0,
$$

satisfies $(\mathrm{C} 1)$ and $(\mathrm{C} 2)$ for the same constant $\alpha$. 
ii). If $\left(X_{t}^{(i)}\right)_{t \geq 0}$ satisfies $(\mathrm{C} 1)$ and $(\mathrm{C} 2)$ for a constant $\alpha_{i} \in(0,2], i=1,2$, then

$$
Z_{t}:=\left(\begin{array}{l}
X_{t}^{(1)} \\
X_{t}^{(2)}
\end{array}\right), \quad t \geq 0,
$$

satisfies (C1) and (C2) with $\alpha:=\min \left\{\alpha_{1}, \alpha_{2}\right\}$.

Proof i). Set $d:=d_{1}=d_{2}$. The characteristic exponent of $\left(Y_{t}\right)_{t \geq 0}$ is $\psi:=\psi^{(1)}+\psi^{(2)}$ where $\psi^{(i)}$ is the characteristic exponent of $X_{t}^{(i)}, i=1$, 2. In particular, we have $\operatorname{Re} \psi \geq \operatorname{Re} \psi^{(1)}$, and therefore the Hartman-Wintner condition (C1) for $\psi^{(1)}$ implies that $\psi$ satisfies $(\mathrm{C} 1)$. Consequently, the law of $Y_{t}$ has a density $p_{t}$ with respect to Lebesgue measure for $t>0$, and it satisfies

$$
p_{t}(x)=\int_{\mathbb{R}^{d}} p_{t}^{(1)}(x-y) \mu_{t}(d y), \quad x \in \mathbb{R}^{d}, t>0,
$$

where $p_{t}^{(1)}$ is the density of $X_{t}^{(1)}$ and $\mu_{t}$ is the law of $X_{t}^{(2)}$. As $p_{t}^{(1)} \in C_{b}^{\infty}\left(\mathbb{R}^{d}\right)$, it follows easily from the differentiation lemma for parameter dependent integrals, see e.g. [35], that

$$
\nabla p_{t}(x)=\int_{\mathbb{R}^{d}} \nabla_{x} p_{t}^{(1)}(x-y) \mu_{t}(d y), \quad x \in \mathbb{R}^{d}, t>0 .
$$

Hence, by Tonelli's theorem and the gradient estimate (C2) for $p_{t}^{(1)}$,

$$
\int_{\mathbb{R}^{d}}\left|\nabla p_{t}(x)\right| d x \leq \int_{\mathbb{R}^{d}} \int_{\mathbb{R}^{d}}\left|\nabla_{x} p_{t}^{(1)}(x-y)\right| d x \mu_{t}(d y) \leq c t^{-1 / \alpha}, \quad t \in(0,1) .
$$

ii). It is obvious that the characteristic exponent $(\xi, \eta) \mapsto \psi^{(1)}(\xi)+\psi^{(2)}(\eta)$ of $\left(Z_{t}\right)_{t \geq 0}$ satisfies $(\mathrm{C} 1)$ whenever $\psi^{(1)}$ and $\psi^{(2)}$ satisfy $(\mathrm{C} 1)$. Since the density of $Z_{t}$ is given by

$$
p_{t}(x, y)=p_{t}^{(1)}(x) p_{t}^{(2)}(y), \quad x \in \mathbb{R}^{d_{1}}, y \in \mathbb{R}^{d_{2}}, t>0
$$

we can follow the reasoning from the first part, i.e. apply the differentiation lemma and Tonelli's theorem, to find that

$$
\int_{\mathbb{R}^{d_{1}+d_{2}}}\left|\nabla p_{t}(z)\right| d z \leq c t^{-1 / \min \left\{\alpha_{1}, \alpha_{2}\right\}}, \quad t \in(0,1) .
$$

Corollary 3 Let $\left(X_{t}\right)_{t \geq 0}$ be a Lévy process with Lévy triplet $(b, Q, v)$. If $Q$ is strictly positive definite, then the interior Schauder estimates in Theorem 1, Corollary 1 and 2 hold with $\alpha=2$.

If there is no jump part, i.e. $v=0$, then the infinitesimal generator of $\left(X_{t}\right)_{t \geq 0}$ is given by

$$
A f(x)=b \cdot \nabla f(x)+\frac{1}{2} \nabla \cdot Q \cdot \nabla f(x), \quad f \in C_{c}^{\infty}\left(\mathbb{R}^{d}\right),
$$

and Corollary 3 yields the classical interior Schauder estimates for solutions to the equation $A f=g$ associated with the second order differential operator $A$, see e.g. [8].

Proof of Corollary 3 The Lévy process $\left(X_{t}\right)_{t \geq 0}$ has a representation of the form

$$
X_{t}=Q W_{t}+J_{t}, \quad t \geq 0,
$$

where $\left(B_{t}\right)_{t \geq 0}$ is a Brownian motion and $\left(J_{t}\right)_{t \geq 0}$ is a Lévy process with Lévy triplet $(b, 0, v)$. Since the transition density of $Q B_{t}$ is of Gaussian type and $Q$ is strictly positive 
definite, it is straightforward to check that $\left(Q B_{t}\right)_{t \geq 0}$ satisfies (C1) and (C2) with $\alpha=2$. The Lévy process $\left(J_{t}\right)_{t \geq 0}$ is independent of $\left(Q B_{t}\right)_{t \geq 0}$, and therefore Proposition $\left.2 \mathrm{i}\right)$ shows that $\left(X_{t}\right)_{t \geq 0}$ satisfies (C1) and (C2) with $\alpha=2$. If we choose $\gamma=2$, then $\int_{|y| \leq 1}|y|^{\gamma} v(d y)<\infty$ and (C3) is trivial. Hence, the assumptions (C1)-(C3) of Theorem 1, Corollary 1 and Corollary 2 are satisfied for $\alpha=\gamma=2$.

Our next result applies to a large class of jump Lévy processes, including stable Lévy processes. It is a direct consequence of the gradient estimates obtained in [34].

Corollary 4 Let $\left(X_{t}\right)_{t \geq 0}$ be a pure-jump Lévy process. Assume that its Lévy measure $v$ satisfies

$$
\nu(B) \geq \int_{0}^{r_{0}} \int_{\mathbb{S}^{d-1}} \mathbb{1}_{B}(r \theta) r^{-1-\varrho} \mu(d \theta) d r, \quad B \in \mathcal{B}\left(\mathbb{R}^{d} \backslash\{0\}\right),
$$

for some constants $r_{0}>0, \varrho \in(0,2)$ and a finite measure $\mu$ on the unit sphere $\mathbb{S}^{d-1} \subseteq \mathbb{R}^{d}$ which is non-degenerate, in the sense that its support is not contained in $\mathbb{S}^{d-1} \cap V$ for some lower-dimensional subspace $V \subseteq \mathbb{R}^{d}$. If

$$
\int_{|y| \leq 1}|y|^{\gamma} v(d y)<\infty
$$

for some $\gamma<1+\varrho$, then the interior Schauder estimates from Theorem 1, Corollary 1 and 2 hold with $\alpha=\varrho$.

If the Lévy measure $v$ is exactly the right-hand side of Eq. 27, then the assumption (28) is trivially satisfied; this is, for instance, the case if $\left(X_{t}\right)_{t \geq 0}$ is isotropic $\alpha$-stable or relativistic $\alpha$-stable. In particular, Corollary 4 generalizes [29, Theorem 1.1].

The next corollary gives a criterion for $(\mathrm{C} 1)-(\mathrm{C} 3)$ in terms of the growth of the characteristic exponent of $\left(X_{t}\right)_{t \geq 0}$.

Corollary 5 Let $\left(X_{t}\right)_{t \geq 0}$ be a Lévy process with infinitesimal generator $(A, \mathcal{D}(A))$. If the characteristic exponent $\psi$ satisfies the sector condition, $|\operatorname{Im} \psi(\xi)| \leq c \operatorname{Re} \psi(\xi)$, and

$$
\operatorname{Re} \psi(\xi) \asymp|\xi|^{\varrho} \quad \text { as }|\xi| \rightarrow \infty
$$

for some $\varrho \in(0,2)$, then the interior Schauder estimates in Theorem 1, Corollary 1 and 2 hold with $\alpha=\varrho$.

Proof The Hartman-Wintner condition (C1) is trivially satisfied. It follows from [34] that the gradient estimate $\int_{\mathbb{R}^{d}}\left|\nabla p_{t}(x)\right| d x \leq c t^{-1 / \varrho}$ holds for $t \in(0,1)$, i.e. $\alpha=\varrho$ in $(\mathrm{C} 2)$. Moreover, Eq. 29 implies that the Lévy measure $v$ satisfies $\int_{|y| \leq 1}|y|^{\beta} v(d y)<\infty$ for all $\beta>\varrho$, cf. [19, Lemma A.2], and by choosing $\beta$ close to $\varrho$, we find that the balance condition (C3) holds.

Corollary 5 covers many important and interesting examples, e.g.

- isotropic stable, relativistic stable and tempered stable Lévy processes,

- subordinated Brownian motions with characteristic exponent $\psi(\xi)=f\left(|\xi|^{2}\right)$ for a Bernstein function $f$ satisfying $f(\lambda) \asymp \lambda \varrho / 2$ for large $\lambda$, cf. [33] for details.

- Lévy processes with symbol of the form

$$
\psi(\xi)=|\xi|^{\varrho}+|\xi|^{\beta}, \quad \xi \in \mathbb{R}^{d},
$$

for $\beta \in(0, \varrho)$. 
In the final part of this section, we discuss the role of the condition (C3) and give examples where the condition fails. First, we would like to explain that (C3) is essentially a balance condition on the growth of $\operatorname{Re} \psi$ at infinity. Let us assume for simplicity that $Q=0$ and that the characteristic exponent $\psi$ satisfies the sector condition $|\operatorname{Im} \psi(\xi)| \leq c \operatorname{Re} \psi(\xi)$, i.e. there is no dominating drift part. It is known that - under these assumptions - the growth of $\operatorname{Re} \psi$ at infinity is closely related to the existence of moments $\int_{|y| \leq 1}|y|^{\gamma} v(d y)$. More precisely, the Blumenthal-Getoor index at infinity

$$
\beta_{\infty}:=\inf \left\{\gamma>0 ; \lim _{r \rightarrow \infty} \frac{1}{r^{\gamma}} \sup _{|\xi| \leq r}|\operatorname{Re} \psi(\xi)|<\infty\right\}
$$

satisfies

$$
\beta_{\infty}=\inf \left\{\gamma>0 ; \int_{|y| \leq 1}|y|^{\gamma} v(d y)<\infty\right\},
$$

cf. [31, Proposition 5.4]. In particular, if $\gamma>0$ is such that $\int_{|y| \leq 1}|y|^{\gamma} v(d y)<\infty$, then $|\operatorname{Re} \psi(\xi)| \leq C(1+|\xi|)^{\gamma+\varepsilon}$ for any $\varepsilon>0$. On the other hand, (C2) means, roughly, that $\operatorname{Re} \psi(\xi) \geq c\left(1+|\xi|^{\alpha}\right)$. For (C3) we need that $|\gamma-\alpha|<1$, that is, the lower and upper growth rate of $\operatorname{Re} \psi$ at infinity should be sufficiently close to each other. Our next examples show that this balance condition may fail if $\psi$ is highly anisotropic or if $\operatorname{Re} \psi$ oscillates at infinity.

Consider the operator

$$
A f(x, y)=-\left(-\frac{\partial^{2}}{\partial x^{2}}\right)^{\beta_{1} / 2} f(x, y)-\left(-\frac{\partial^{2}}{\partial y^{2}}\right)^{\beta_{2} / 2} f(x, y), \quad f \in C_{c}^{\infty}\left(\mathbb{R}^{2}\right), x, y \in \mathbb{R},
$$

which arises as infinitesimal generator of a Lévy process $\left(X_{t}\right)_{t \geq 0}$ of the form $X_{t}=$ $\left(X_{t}^{(1)}, X_{t}^{(2)}\right)$ where $\left(X_{t}^{(i)}\right)_{t \geq 0}$ are independent one-dimensional isotropic stable Lévy processes with index $\beta_{i} \in(0,2], i=1,2$, see e.g. [28] for more information. The difference $\left|\beta_{1}-\beta_{2}\right|$ measures how much the behaviour of the first coordinate $\left(X_{t}^{(1)}\right)_{t \geq 0}$ differs from the behaviour of the second coordinate $\left(X_{t}^{(2)}\right)_{t \geq 0}$. If $\left|\beta_{1}-\beta_{2}\right|$ is large (i.e. close to 2), we are dealing with a highly anisotropic process.

Example 1 Let $\left(X_{t}\right)_{t \geq 0}$ be a two-dimensional Lévy process with characteristic exponent

$$
\psi(\xi, \eta)=|\xi|^{\beta_{1}}+|\eta|^{\beta_{2}}, \quad \xi, \eta \in \mathbb{R}
$$

for some constants $\beta_{i} \in(0,2], i=1,2$.

i). The balance condition (C3) holds if, and only if, $\left|\beta_{1}-\beta_{2}\right|<1$.

ii). If $\left|\beta_{1}-\beta_{2}\right|<1$ then the interior Schauder estimates in Theorem 1, Corollary 1 and Corollary 2 hold with $\alpha=\min \left\{\beta_{1}, \beta_{2}\right\}$.

Let us mention a further class of processes illustrating the role of the balance condition (C3). Farkas et al. [7, Example 1.1.15] showed that for every $0<\beta<\alpha<2$ there exists a onedimensional Lévy process whose characteristic exponent $\psi$ oscillates for $|\xi| \rightarrow \infty$ between $|\xi|^{\beta}$ and $2|\xi|^{\alpha}$. Since the growth of $\psi$ at infinity is closely linked to existence of fractional moments $\int_{|y| \leq 1}|y|^{\gamma} v(d y)$, cf. Eq. 30, we have $\int_{|y| \leq 1}|y|^{\gamma} v(d y)<\infty$ only for $\gamma>\beta$ and $\int_{\mathbb{R}^{d}}\left|\nabla p_{t}(x)\right| d x \leq M t^{-1 / \alpha}$. In particular, the balance condition (C3) fails if $\alpha-\beta>1$.

Acknowledgments I am grateful to René Schilling for valuable comments which helped to improve the presentation of this paper. 
Funding Open Access funding enabled and organized by Projekt DEAL.

Open Access This article is licensed under a Creative Commons Attribution 4.0 International License, which permits use, sharing, adaptation, distribution and reproduction in any medium or format, as long as you give appropriate credit to the original author(s) and the source, provide a link to the Creative Commons licence, and indicate if changes were made. The images or other third party material in this article are included in the article's Creative Commons licence, unless indicated otherwise in a credit line to the material. If material is not included in the article's Creative Commons licence and your intended use is not permitted by statutory regulation or exceeds the permitted use, you will need to obtain permission directly from the copyright holder. To view a copy of this licence, visit http://creativecommonshorg/licenses/by/4.0/.

\section{Appendix: Proof of Lemma 1}

For the proof of our results we used the following lemma, which was already stated in Section 2.

Lemma 5 Let $\beta \in(0, \infty)$, and let $U \subseteq \mathbb{R}^{d}$ be open. The following statements hold for any $j \geq k:=\lfloor\beta\rfloor+1$ :

i). There exists a constant $c>0$ such that

$$
\sup _{0<|h| \leq r} \frac{\left|\Delta_{h}^{k} f(x)\right|}{|h|^{\beta}} \leq c r^{-\beta}\|f\|_{\infty, U}+c \sup _{0<|h| \leq r / j} \sup _{z \in B(x, r(k+1))} \frac{\left|\Delta_{h}^{j} f(z)\right|}{|h|^{\beta}}
$$

for all $f \in C_{b}(U), r>0$, and $x \in U$ with $B(x, r(k+2)) \subseteq U$.

ii). If $\beta>1$ then there exists a constant $c>0$ such that

$$
\max _{i=1, \ldots, d}\left|\partial_{x_{i}} f(x)\right| \leq c r^{-\beta}\|f\|_{\infty, U}+c \sup _{0<|h| \leq r / j} \sup _{z \in B(x, r(k+1))} \frac{\left|\Delta_{h}^{j} f(z)\right|}{|h|^{\beta}}
$$

for all $f \in C_{b}^{1}(U), r>0$ and $x \in U$ with $B(x, r(k+2)) \subseteq U$.

Proof First of all, we note that it suffices to prove both statements for $f \in C_{b}^{2}(U)$; the inequalities can be extended using a standard approximation technique, e.g. by considering $f_{i}:=f * \varphi_{i}$ for a sequence of mollifiers $\left(\varphi_{i}\right)_{i \geq 1}$.

Denote by $\tau_{h} f(x):=f(x+h)$ the shift operator. A straightforward computation shows that

$$
\Delta_{h}^{n}(u \cdot v)=\sum_{\ell=0}^{n}\left(\begin{array}{l}
n \\
\ell
\end{array}\right) \Delta_{h}^{\ell} u \cdot \Delta_{h}^{n-\ell} \tau_{h}^{\ell} v
$$

holds for any $n \in \mathbb{N}, h \in \mathbb{R}^{d}$ and any two functions $u, v$.

To prove i) we note that the assertion is obvious for $j=k$, and so it suffices to consider $j>k$. We will first establish the following auxiliary statement: There exists a constant $C>0$ such that

$$
\sup _{0<|t| \leq 1} \frac{\left|\Delta_{t}^{k} g(0)\right|}{|t|^{\beta}} \leq C\|g\|_{\infty,(-k-2, k+2)}+C \sup _{0<|t| \leq 1 / j} \sup _{|y| \leq k+1} \frac{\left|\Delta_{t}^{j} g(y)\right|}{|t|^{\beta}}
$$

for any twice differentiable bounded function $g:(-k-2, k+2) \rightarrow \mathbb{R}$. To this end, pick $\chi \in C_{c}^{\infty}(\mathbb{R})$ such that $\mathbb{1}_{[-k, k]} \leq \chi \leq \mathbb{1}_{(-k-1 / 3, k+1 / 3)}$. Clearly,

$$
\sup _{0<|t| \leq 1} \frac{\left|\Delta_{t}^{k} g(0)\right|}{|t|^{\beta}}=\sup _{0<|t| \leq 1} \frac{\left|\Delta_{t}^{k}(g \chi)(0)\right|}{|t|^{\beta}} .
$$


Using the equivalence of the norms on $\mathcal{C}_{b}^{\beta}(\mathbb{R})$, cf. Eq. 7, we get

$$
\begin{aligned}
& \sup _{0<|t| \leq 1} \frac{\left|\Delta_{t}^{k} g(0)\right|}{|t|^{\beta}} \leq\|g \chi\|_{\mathcal{C}_{b}^{\beta}(\mathbb{R})} \leq c_{1}\|g \chi\|_{\infty}+c_{1} \sup _{|t| \leq 1} \sup _{y \in \mathbb{R}} \frac{\left|\Delta_{t}^{2 j}(g \chi)(y)\right|}{|t|^{\beta}} \\
& \leq c_{1}^{\prime}\|g\|_{\infty,(-k-1, k+1)}+c_{1} \sup _{|t| \leq 1 /(6 j)} \sup _{y \in \mathbb{R}} \frac{\left|\Delta_{t}^{2 j}(g \chi)(y)\right|}{|t|^{\beta}}
\end{aligned}
$$

for some constants $c_{1}$ and $c_{1}^{\prime}$. As $\chi=0$ on $\mathbb{R} \backslash(-k-1 / 3, k+1 / 3)$, we have $\Delta_{t}^{2 j} g(y)=0$ for all $|y|>k+\frac{2}{3}$ and $|t| \leq \frac{1}{6 j}$. Consequently,

$$
\sup _{0<|t| \leq 1} \frac{\left|\Delta_{t}^{k} g(0)\right|}{|t|^{\beta}} \leq c_{1}^{\prime}\|g\|_{\infty,(-k-1, k+1)}+c_{1} \sup _{|t| \leq 1 /(6 j)} \sup _{|y| \leq k+2 / 3} \frac{\left|\Delta_{t}^{2 j}(g \chi)(y)\right|}{|t|^{\beta}} .
$$

Since

$$
\left|\Delta_{t}^{\ell} g(y)\right|=\left|\Delta_{t}^{\ell-j} \Delta_{t}^{j} g(y)\right| \leq 2^{\ell-j} \sup _{|z-y| \leq(\ell-j) t}\left|\Delta_{t}^{j} g(z)\right|, \quad \ell \geq j
$$

and

$$
\left\|\Delta_{t}^{\ell} \chi\right\|_{\infty} \leq c|t|^{k}\|\chi\|_{C_{b}^{k}(\mathbb{R})}, \quad \ell \geq j \geq k
$$

an application of the product formula (33) gives

$$
\begin{aligned}
\left|\Delta_{t}^{2 j}(g \chi)(y)\right| & \leq c_{2}\|g\|_{\infty,(-k-1, k+1)} \sum_{\ell=0}^{j-1}\left\|\Delta_{t}^{2 j-\ell} \chi\right\|_{\infty}+c_{2}\|\chi\|_{\infty} \sum_{\ell=j}^{2 j}\left|\Delta_{t}^{\ell} g(y)\right| \\
& \leq c_{3}\|g\|_{\infty,(-k-1, k+1)}|t|^{k}\|\chi\|_{C_{b}^{k}(\mathbb{R})}+c_{3} \sup _{|z-y| \leq 2 t j}\left|\Delta_{t}^{j} g(z)\right|
\end{aligned}
$$

for all $|y| \leq k+\frac{2}{3}$ and $|t| \leq \frac{1}{6 j}$. Combining this estimate with Eq. 35 and noting that $\beta \leq k$ proves (34). Now if $f \in C_{b}^{2}(U)$, then we apply (34) with $g(t):=f(x+r t h)$ for fixed $|h|=1$ to get the desired inequality.

It remains to prove ii). First we consider the case $\beta \in(1,2)$ and $j=2$. The auxiliary inequality which we need is

$$
\left|g^{\prime}(0)\right| \leq C\|g\|_{\infty,(-3,3)}+C \sup _{|t| \leq 1 / 2} \sup _{|y| \leq 2} \frac{\left|\Delta_{t}^{2} g(y)\right|}{|t|^{\beta}}
$$

for a uniform constant $C>0$ where $g: \mathbb{R} \rightarrow \mathbb{R}$ is differentiable on $(-3,3)$. To this end,

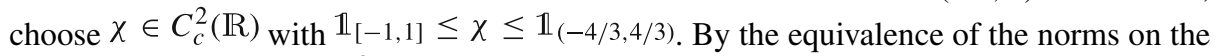
Hölder-Zygmund space $\mathrm{e}_{b}^{\beta}(\mathbb{R})$, cf. Eq. 7, we get

$$
\left|g^{\prime}(0)\right|=\left|(g \chi)^{\prime}(0)\right| \leq\|g \chi\|_{C_{b}^{1}(\mathbb{R})} \leq\|g \chi\|_{\mathcal{C}_{b}^{\beta}(\mathbb{R})} \leq c_{4}\|g \chi\|_{\infty}+c_{4} \sup _{|t| \leq 1} \sup _{y \in \mathbb{R}} \frac{\left|\Delta_{t}^{4}(g \chi)(y)\right|}{|t|^{\beta}}
$$

for some finite constant $c_{4}>0$. Following the reasoning in the first part of the proof (with $k=1$ and $j=2$ ) yields (36). Applying (36) for $g(t):=f\left(x+r t e_{j}\right)$, where $e_{j}$ is the $j$-th unit vector in $\mathbb{R}^{d}$, gives ii) for $\beta \in(1,2)$ and $j=2$. In combination with i), this yields the desired inequality for every $\beta>1$ and $j \geq\lfloor\beta\rfloor+1$. 


\section{References}

1. Bass, R.F.: Regularity results for stable-like operators. J. Funct. Anal. 259, 2693-2722 (2009)

2. Bouleau, N., Hirsch, F.: Dirichlet Forms and Analysis on Wiener Space. De Gruyter (1991)

3. Dellacherie, C., Meyer, P.-A.: Théorie du Potentiel Associée à une Résolvante - Théorie des Processus de Markov. Hermann, Paris (1987)

4. de Raynal, P., Menozzi, S., Priola, E.: Schauder estimates for drifted fractional operators in the supercritical case. J. Funct. Anal. 128. https://doi.org/10.1016/j.jfa.2019.108425 (2020)

5. Dong, H., Kim, D.: Schauder estimates for a class of non-local elliptic equations. Discrete Contin. Dyn. Syst. 33, 2319-2347 (2012)

6. Fall, M.M., Weth, T.: Liouville theorems for a general class of nonlocal operators. Potential Anal. 45, 187-200 (2016)

7. Farkas, W., Jacob, N., Schilling, R.L.: Function spaces related to continuous negative definite functions: $\psi$-Bessel potential spaces. Diss. Math. 393, 1-62 (2001)

8. Gilbarg, D., Trudinger, N.S.: Elliptic Partial Differential Equations of Second Order. Springer, Berlin (1983)

9. Grzywny, T., Kwaśnicki, M.: Potential kernels, probabilities of hitting a ball, harmonic functions and the boundary Harnack inequality for unimodal Lévy processes. Stoch. Proc. Appl. 128, 1-38 (2018)

10. Hansen, W.: Intrinsic Hölder continuity of harmonic functions. Potential Anal. 47, 1-12 (2017)

11. Hao, Z., Wang, Z., Wu, M.: Schauder's estimates for nonlocal equations with singular Lévy measures. Preprint, arXiv:2002.09887 (2020)

12. Imbert, C., Jin, T., Shvydkoy, R.: Schauder estimates for an integro-differential equation with applications to a nonlocal Burgers equation. Ann. Fac. Sc. Toulouse 27, 667-677 (2018)

13. Jacob, N.: Pseudo-differential Operators and Markov Processes. Akademie (1996)

14. Jacob, N.: Pseudo Differential Operators and Markov Processes II. Imperial College Press/World Scientific (2002)

15. Jacob, N.: Pseudo Differential Operators and Markov Processes III. Imperial College Press/World Scientific (2005)

16. Khoshnevisan, D., Schilling, R.L.: From Lévy-type Processes to Parabolic SPDEs. Birkhäuser, Basel (2016)

17. Knopova, V., Schilling, R.L.: A note on the existence of transition probability densities of Lévy processes. Forum. Math. 25, 125-149 (2013)

18. Kühn, F.: Schauder estimates for equations associated with Lévy generators. Integral Equ. Oper. Theory 91, 10 (2019)

19. Kühn, F., Schilling, R.L.: On the domain of fractional Laplacians and related generators of Feller processes. J. Funct. Anal. 276, 2397-2439 (2019)

20. Kühn, F.: Schauder estimates for Poisson equations associated with non-local Feller generators. Preprint arXiv:1902.01760. To appear: J. Theor. Probab. (2020)

21. Kühn, F.: A Liouville theorem for Lévy generators. Preprint, arXiv:2001.02528. To appear: Positivity (2020)

22. Kulczycki, T., Ryznar, M.: Gradient estimates of harmonic functions and transition densities for Lévy processes. Trans. Am. Math. Soc. 368, 281-318 (2015)

23. Kwaśnicki, M.: Ten equivalent definitions of the fractional Laplace operator. Fract. Calc. Anal. Appl. 20, 7-51 (2017)

24. Liang, M., Wang, J.: Spatial regularity of semigroups generated by Lévy type operators. Math. Nachr. 292, 1551-1566 (2019)

25. Lunardi, A.: Schauder theorems for linear elliptic and parabolic problems with unbounded coefficients in $\mathbb{R}^{n}$. Studia Math. 128, 171-198 (1998)

26. Mikulevicius, R., Pragarauskas, H.: On the Cauchy problem for integro-differential operators in Hölder classes and the uniqueness of the martingale problem. Potential Anal. 40, 539-563 (2014)

27. Priola, E.: Davie's type uniqueness for a class of SDEs with jumps. Ann. Inst. Henri Poincaré Probab. Stat. 54, 694-725 (2018)

28. Pruitt, W.E., Taylor, S.J.: Sample path properties of processes with stable components. Z. Wahrscheinlichkeitstheorie Verw. Gebiete 12, 267-289 (1969)

29. Ros-Oton, X., Serra, J.: Regularity theory for general stable operators. J. Differ. Equ. 260, 8675-8715 (2016)

30. Sato, K.: Lévy Processes and Infinitely Divisible Distributions. Cambridge University Press, Cambridge (2013) 
31. Schilling, R.L.: Growth and Hölder conditions for the sample paths of Feller processes. Probab. Theory Relat. Fields 112, 565-611 (1998)

32. Schilling, R.L.: Function spaces as path spaces of Feller processes. Math. Nachr. 217, 147-174 (2000)

33. Schilling, R.L., Song, R., Vondraček, Z. Bernstein Functions: Theory and Applications, 2nd edn. De Gruyter, Berlin (2012)

34. Schilling, R.L., Sztonyk, P., Wang, J.: Coupling property and gradient estimates of Lévy processes via the symbol. Bernoulli 18, 1128-1149 (2012)

35. Schilling, R.L. Measures, Integrals and Martingales, 2nd edn. Cambridge University Press, Cambridge (2017)

36. Stein, E.M.: Singular Integrals and Differentiability Properties of Functions. Princeton University Press, Princeton (1970)

37. Sztonyk, P.: Regularity of harmonic functions for anisotropic fractional Laplacians. Math. Nachr. 283, 289-311 (2010)

38. Triebel, H.: Interpolation Theory, Function Spaces, Differential Operators. North-Holland, New York (1978)

39. Zhang, X., Zhao, G.: Dirichlet problem for supercritical non-local operators. Preprint, arXiv:1809.05712 (2018)

Publisher's Note Springer Nature remains neutral with regard to jurisdictional claims in published maps and institutional affiliations. 